\title{
Reliable Computation of Robust Response Tori on the Verge of Breakdown*
}

\author{
Jordi-Lluís Figueras ${ }^{\dagger}$ and Àlex Haro ${ }^{\dagger}$
}

\begin{abstract}
We prove the existence and local uniqueness of invariant tori on the verge of breakdown for two systems: the quasi-periodically driven logistic map and the quasi-periodically forced standard map. These systems exemplify two scenarios: the Heagy-Hammel route for the creation of strange nonchaotic attractors and the nonsmooth bifurcation of saddle invariant tori. Our proofs are computerassisted and are based on a tailored version of the Newton-Kantorovich theorem. The proofs cannot be performed using classical perturbation theory because the two scenarios are very far from the perturbative regime, and fundamental hypotheses such as reducibility or hyperbolicity either do not hold or are very close to failing. Our proofs are based on a reliable computation of the invariant tori and a careful study of their dynamical properties, leading to the rigorous validation of the numerical results with our novel computational techniques.
\end{abstract}

Key words. normally hyperbolic invariant manifolds, computer validations, invariant tori, strange nonchaotic attractors

AMS subject classifications. 37C55, 37D10, 65P99

DOI. $10.1137 / 100809222$

1. Introduction. The goal of this paper is to present a new methodology to provide rigorous proofs of the existence and local uniqueness of (fiberwise hyperbolic) invariant tori in quasi-periodic systems, even in cases in which the available perturbative theory does not apply. The methodology is suitable for computer-assisted proofs and consists in checking the hypotheses of a validation result based on the Newton-Kantorovich theorem [27]. As an application of the methodology, we prove the existence and local uniqueness of invariant tori on the verge of breakdown in two scenarios: the Heagy-Hammel route to strange nonchaotic attractors (SNA) [32] in a quasi-periodically driven logistic map and the breakdown of saddle tori [26] in a quasi-periodically forced standard map.

Organization of the paper. In this introductory section we present an overview of the paper, including the rigorous validating results of existence of invariant tori in several examples and a brief discussion of the methodology. In section 2 we summarize the theoretical framework necessary for the computer-assisted proofs and present a validation algorithm. In section 3 we present the Fourier models for the rigorous manipulation of Fourier approximations (see also Appendix A) and the implementation of the validation algorithm. Sections 4 and 5 report the proofs of the rigorous validations which have to be carried out after accurate numerical computations.

\footnotetext{
${ }^{*}$ Received by the editors September 20, 2010; accepted for publication (in revised form) by B. Krauskopf December 4, 2011; published electronically April 12, 2012. This research was supported by CIRIT 2009SGR-67 and MCNN MTM2009-09723.

http://www.siam.org/journals/siads/11-2/80922.html

${ }^{\dagger}$ Departament de Matemàtica Aplicada i Anàlisi, Universitat de Barcelona, Gran Via 585, 08007 Barcelona, Spain (figueras@maia.ub.es, alex@maia.ub.es). The work of the first author was supported by Departament d'Universitats, Recerca i Societat de la Informació de la Generalitat de Catalunya.
} 
1.1. Robust response tori in quasi-periodic systems. The long-term behavior of a dynamical system is organized by its invariant objects. Hence, it is important to identify the robust invariant objects that persist under perturbations of the system. In applications, we can produce numerical approximations of these objects and we may wish to turn the nonrigorous calculations into theorems. Hence, the question is to establish whether a numerical approximation persists as an invariant object of the dynamical system and to provide rigorous error bounds.

In this paper, we address this question for a particular class of dynamical systems and invariant objects. The systems we consider are quasi-periodically forced, that is, coupled with an irrational rotation, and the invariant objects are invariant tori carrying this irrational rotation. These tori are the response to the quasi-periodic forcing and are geometrically described as graphs of the state variables over the coupled angles describing the quasi-periodic motion [59]. Since it has been known for a long time that persistence of invariant manifolds is closely related to the concept of normal hyperbolicity $[21,34,50,57]$, here we consider the analogous concept, tailored for skew products over rotations. Roughly speaking, an invariant torus is fiberwise hyperbolic if the linearized dynamics on the normal bundle is exponentially dichotomous, that is, the normal bundle splits into stable and unstable bundles on which the dynamics is uniformly contracting and expanding, respectively. Notice that the tangent dynamics is dominated by the normal dynamics, since the former presents zero Lyapunov exponents. This implies that fiberwise hyperbolic invariant tori are robust and are as smooth as the system [28].

Most of the results regarding the existence of invariant objects in the literature are in fact perturbative $[21,34,50,57]$ and provide rather pessimistic estimates of the persistence of the invariant objects when applied to concrete examples. In this paper we adopt the functional framework described in [27], which leads to an a posteriori result based on the Newton-Kantorovich theorem. Hence, the rigorous validation of numerical computations consists in checking the hypotheses of this theorem. Notably, the applicability of Newton's method for computing response tori is related with the property of fiberwise hyperbolicity. The methodology is suitable for validating invariant tori that are very close to breakdown.

1.2. Reliable computations on the verge of breakdown. The transition from regular to irregular motion is a difficult mathematical problem which arises in various fields, such as solid state physics, chemical reaction dynamics, climate dynamics, and neuroscience. In systems under quasi-periodic forcing, the transition can be understood as the phenomenon of breakdown of response invariant tori. A main problem is providing rigorous bounds of the parameters for which smooth invariant tori do exist, close to the estimated thresholds of breakdown, since we may need rigorous delimitations of the boundaries between the regular motion and the irregular motion. In this paper we report the application of computer-assisted proofs of the existence of invariant tori in two scenarios: the Heagy-Hammel route to an SNA [32] and the nonsmooth breakdown of saddle invariant tori [26].

1.2.1. Rigorous validations in the Heagy-Hammel route. In quasi-periodic dissipative systems, it has been observed that an attracting smooth torus may nonsmoothly bifurcate into an attracting object of complicated geometry (not even continuous) but still carry a nonchaotic (in fact quasi-periodic) dynamics, the SNA. The discovery of this extremely interesting behavior [25] (see also [33]) produced an explosion of numerical and experimental studies re-

Copyright (c) by SIAM. Unauthorized reproduction of this article is prohibited. 
porting mechanisms for the birth of SNA which still resonates today (see, e.g., [22, 55] and references therein). Further theoretical studies with rigorous explanations and mathematical proofs of some of these mechanisms have been considered in the mathematical literature (see, e.g., $[6,7,30,35,40,60,61])$, and all of them involve the collision of invariant tori. The Heagy-Hammel route [32] falls into this category.

In the Heagy-Hammel route, a period 2 attracting torus (born in a period doubling bifurcation) collides with its companion repelling torus, producing an SNA. In this transition, the repelling torus is preserved, while the period 2 attracting torus is destroyed. This situation has been observed in numerical experiments on the following quasi-periodically driven logistic map:

$$
\left\{\begin{array}{l}
z_{n+1}=a\left(1+D \cos \left(2 \pi \theta_{n}\right)\right) z_{n}\left(1-z_{n}\right), \\
\theta_{n+1}=\theta_{n}+\omega, \quad \bmod 1,
\end{array}\right.
$$

where $\omega=\frac{1}{2}(\sqrt{5}-1)$ and $a$ and $D$ are parameters.

In the following, we fix $D=0.1$ and let $a>0$ vary. Numerical experiments (see subsection section 4.1 for further details) show that for $a \in] a_{\mathrm{p}}, a_{\mathrm{c}}$ [, with $a_{\mathrm{p}} \simeq 3.141875$ and $a_{\mathrm{c}} \simeq 3.271383$, there is a period 2 attracting torus. See Figure 1. This period 2 attracting torus is born in a period doubling bifurcation at $a=a_{\mathrm{p}}$, and it is destroyed at $a=a_{\mathrm{c}}$ when colliding with the repelling torus. The repelling torus survives the collision and in fact exists for $a \in] a_{\mathrm{p}},+\infty[$.

In this scenario, a key role is played by the noninvertibility of (1.1). Interestingly, the role of noninvertibility in global bifurcations was already noted in $[1,2]$. In the present example, the linearized dynamics of the period 2 attracting torus can be either reducible (which means that it is invertible) or nonreducible. The linearized dynamics degenerates when the torus crosses the critical curve $\left\{z=\frac{1}{2}\right\}$, at $a_{\mathrm{r}} \simeq 3.17496$. When parameter $a$ approaches the threshold $a_{\mathrm{c}}$, the unstable dynamics around the repelling torus must be more apparent on the period 2 attracting torus, since both objects approach each other. In the threshold $a_{\mathrm{c}}$, the closure of the SNA must contain some repelling orbits [60]. Hence, even though the normal dynamics around the period 2 attracting torus is attracting on average (the Lyapunov exponent is negative), it may be locally expanding. This local expansivity and the degeneracy of the linear dynamics are a major drawback in the rigorous validation of the period 2 attracting curve for $a$ close to $a_{\mathrm{c}}$.

The following proposition asserts that the computations of invariant tori in Figure 1 are reliable. In particular, the period 2 attracting torus exists up to a relative distance which is less than $7.3 \cdot 10^{-4}$ of the (numerically) estimated value of the breakdown. This proposition is proved in section 4 .

Proposition 1.1. Consider the skew product (1.1) with $D=0.1$.

(i) For the range of parameters $a \in(3.157065, \infty)$ there exists a continuous family of invariant repellor curves.

(ii) For every parameter $a=3.265,3.268,3.269$ there exists a locally unique period 2 invariant attracting curve.

1.2.2. Rigorous validations on the verge of a hyperbolicity breakdown. We present reliable computations and validations of saddle invariant tori and their invariant stable and unstable bundles in quasi-periodically forced systems. We consider the case in which saddle tori break nonsmoothly (in a sort of nonsmooth Hamiltonian saddle-node bifurcation).

Copyright (c) by SIAM. Unauthorized reproduction of this article is prohibited. 


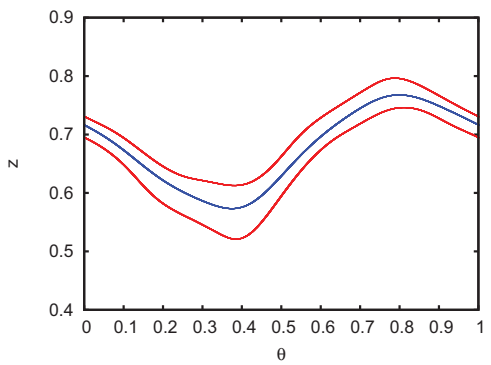

(a) $a=3.160:$ Reducible period 2 torus

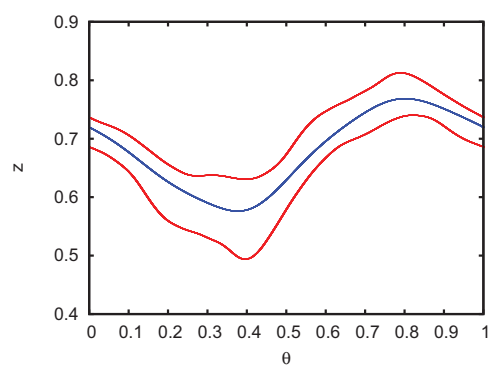

(c) $a=3.180$ : Irreducible period 2 torus

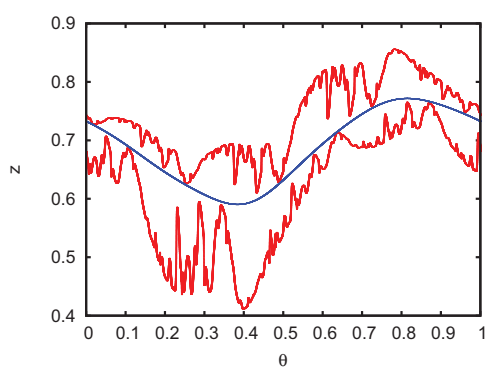

(e) $a=3.269$ : Close to breakup

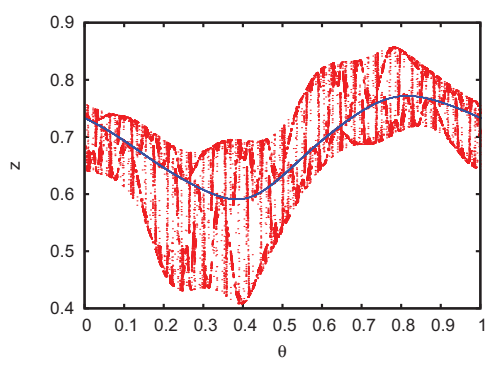

(g) $a=3.272:$ SNA

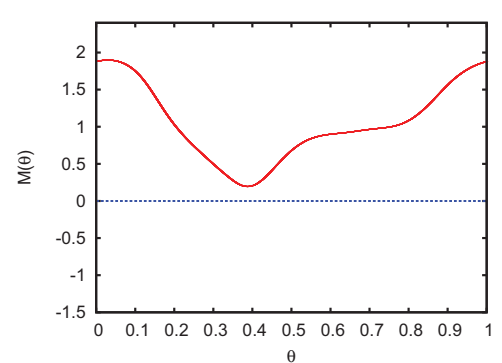

(b) $a=3.160$ : Linearized dynamics of the reducible period 2 torus

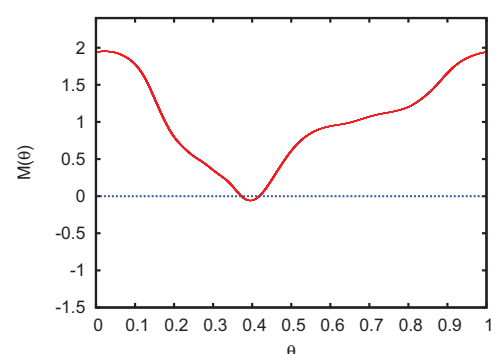

(d) $a=3.180$ : Linearized dynamics of the irreducible period 2 torus

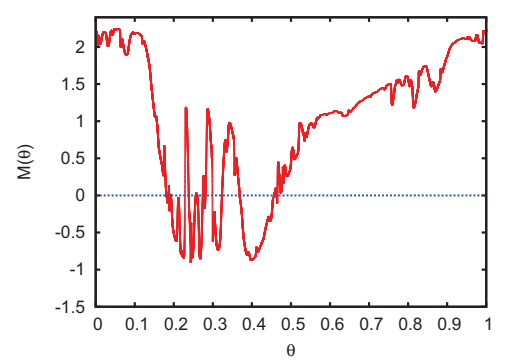

(f) $a=3.269$ : Linearized dynamics of the period 2 torus close to breakup

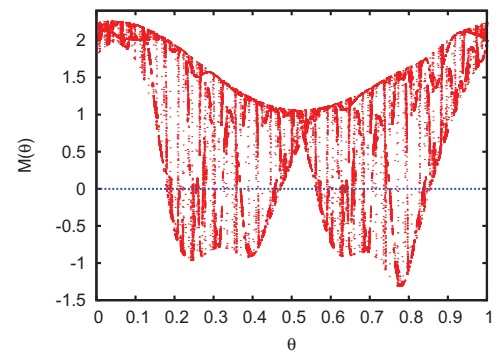

(h) $a=3.272$ : Linearized dynamics of the SNA

Figure 1. On the left, period 2 attracting tori (red) and repelling tori (blue) in the Heagy-Hammel route. On the right, the linearized dynamics of the period 2 attracting tori.

Copyright ( $\odot$ by SIAM. Unauthorized reproduction of this article is prohibited. 
The phenomenon of breakdown of saddle invariant tori in quasiperiodic conservative systems is poorly understood and, as far as we know, only two numerical studies have reported mechanisms and conjectured a theoretical framework [26, 29]. The phenomenon consists in the nonuniform approach to the stable and unstable bundles, deteriorating as the parameters approach a certain threshold. In other words, the projectivizations of the invariant bundles show the typical collision mechanism of creation of SNA observed, e.g., in the Harper map [30, 41]. Moreover, the corresponding Lyapunov multipliers are away from 1. As suggested in $[29,26]$, the nonuniform collision of the bundles leads to a lack of uniform hyperbolicity in the torus and to its breakdown when the parameters cross the threshold.

The model we consider in the present paper is the quasi-periodically forced standard map, defined as

$$
\left\{\begin{array}{l}
x_{n+1}=x_{n}+y_{n+1}, \\
y_{n+1}=y_{n}-\frac{\kappa}{2 \pi} \sin \left(2 \pi x_{n}\right)-\varepsilon \sin \left(2 \pi \theta_{n}\right), \\
\theta_{n+1}=\theta_{n}+\omega, \quad \bmod 1,
\end{array}\right.
$$

where we fix $\omega=\frac{1}{2}(\sqrt{5}-1)$, and $\kappa, \varepsilon$ are parameters.

In the following, we fix $\kappa=1.3$ and let $\varepsilon$ vary. For $\varepsilon=0$ there exists an invariant saddle torus. Numerical experiments (see subsection 5.1 for further details) suggest that there is a limiting value $\varepsilon_{c} \approx 1.2352755$, the critical parameter value, where the saddle torus breaks up: its invariant stable and unstable bundles collide in a nonsmooth manner while the maximal Lyapunov multiplier remains far from zero.

In the nonsmooth breakdown scenario, for $\kappa=1.3$, we prove the existence of the saddle torus up to a bound that is at a relative distance less than $4.3 \cdot 10^{-7}$ from the estimated threshold of breakdown. This is part of the following proposition.

Proposition 1.2. Consider the skew product (1.2) with $\kappa=1.3$ :

(i) For the parameters $\varepsilon=10^{-2} j, j=0, \ldots, 123$, and $\varepsilon=1.231,1.232,1.233,1.234$, $1.235,1.2351,1.2352$ there exist locally unique invariant saddle curves.

(ii) For the range of parameters $\varepsilon \in[0,1.167434]$ there exists a continuous family of invariant saddle curves.

(iii) For every parameter $\varepsilon=1.235270,1.235273,1.235275$ there exists a locally unique invariant saddle curve.

In this bifurcation the computation of invariant tori and their stable and unstable bundles is difficult, since these objects are highly deteriorated and close to breakdown, and simple iteration algorithms of computation do not apply. Notice we have validated Figures 2(c) and 2(d), in which the bundles present SNA-like behavior.

1.3. The methodology. Our methodology represents an advance on the results, numerical algorithms, and experiments presented in [27, 28, 29], which are the inspiration for this paper. In these references, the dynamical characterization of the condition of invariance of a torus leads to a functional equation that fits in the framework of the Newton-Kantorovich theorem [38]; see Theorem 1 in [27] (Theorem 2.8 in the present paper). We emphasize that the nondegeneracy conditions of the Newton-Kantorovich theorem correspond to hyperbolicity properties of the approximate invariant tori. Therefore, starting with an approximate solution of the invariance equation and an approximation of the hyperbolicity properties (i.e., of the stable

Copyright (c) by SIAM. Unauthorized reproduction of this article is prohibited. 


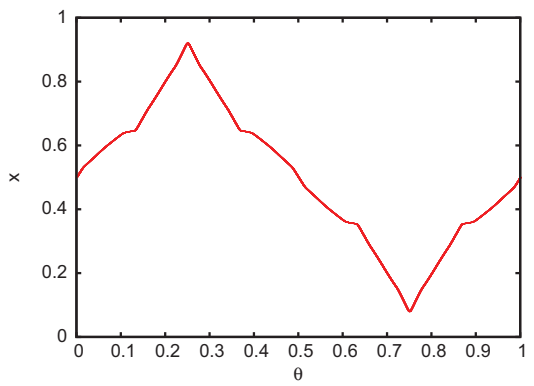

(a) $x$-coordinate projection of the invariant torus. $\varepsilon=1.235$.

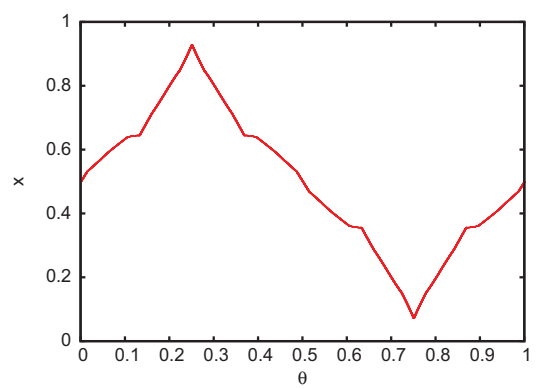

(c) $x$-coordinate projection of the invariant torus. $\varepsilon=1.235275$.

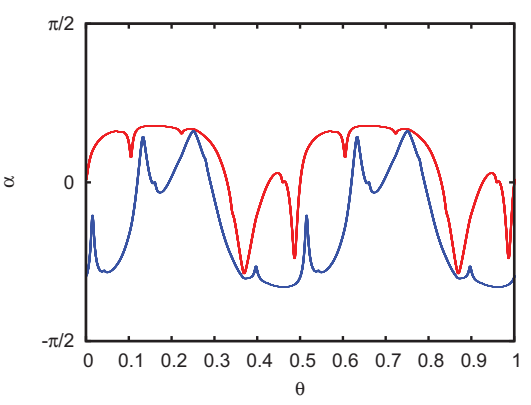

(b) Invariant subbundles. $\varepsilon=1.235$.

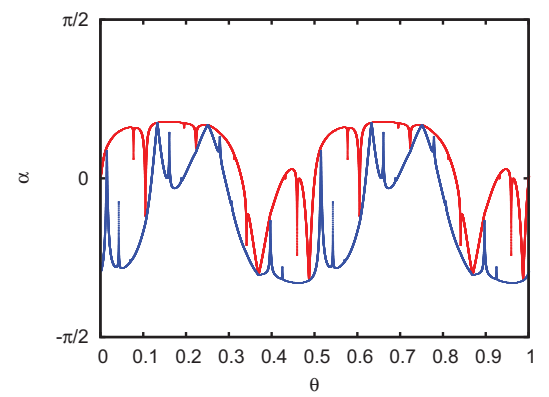

(d) Invariant subbundles. $\varepsilon=1.235275$.

Figure 2. Nonsmooth bifurcation: invariant torus and its subbundles for $\kappa=1.3$ near the bifurcation value $\varepsilon_{c} \approx 1.2352755$. See text for further details.

and unstable bundles), one can use rigorous interval arithmetic [53, 39] to verify the hypothesis of the constructive existence theorem (see Theorem 2.8), which consists in checking several a posteriori bounds. The verification of these bounds leads to the proof of the existence (and local uniqueness) of a true solution of the invariance equation and hence of the true invariant torus and its stable and unstable bundles. In the situations explained in this paper, in which tori are about to break, having accurate and efficient numerical methods is essential to be able to produce approximations that pass the validation test. In this paper we use Fourier methods [29] and rational approximation of frequencies (computing periodic orbits of approximate periodically forced systems) to obtain these approximate solutions. These numerical methods are tailored for the specific class of invariant tori we consider. (See, e.g., [8, 9] for general numerical methods to compute normally hyperbolic invariant manifolds.) An alternative topological approach for validating the existence of invariant sets of normally hyperbolic type has been considered in [11], which is based on the method of covering relations [65]. These methods work for more general dynamical systems but cannot be used to prove the (local) uniqueness of the invariant sets. Moreover, the properties of normal hyperbolicity are checked by using cone conditions, which are extremely difficult to verify for the examples considered here. Our functional analysis flavor for computer-assisted proofs in dynamical systems problems using Newton-like methods in fact has a long history that goes back to the proof of the Feigenbaum 
conjecture in unimodal maps $[45,46]$, the proof of the universality in the period-doubling cascade for area-preserving maps [20], and the proof of the existence of critical invariant tori in Hamiltonian systems [43]. See also the inspiring chapter 7 in [17] and the review [44]. Also, the recent papers $[23,4]$ deal with rigorous computer-assisted validations of zeros of nonlinear functionals defined in infinite dimensional Banach spaces in the context of evolution equations. In these references, the linearizations of the corresponding functionals are compact operators, which have a relatively simple spectrum, making it relatively easy to verify the nondegeneracy conditions required to apply a Newton-like method. In contrast, the operators arising in the problems presented here are noncompact and their spectra are sets of annuli centred at 0 $[51,15]$ (the inner annulus could be a disk if the dynamical system is nonreversible), which makes the (direct) computation of spectra relatively difficult. Fortunately, checking the applicability of Newton's method is equivalent to checking that 1 is not in the spectrum, and this is just rephrasing the condition of hyperbolicity. In this paper, a natural Banach space for use in the parameterizations of tori and their bundles is the space of continuous periodic functions. A suitable method for rigorously enclosing continuous periodic functions is to use (truncated) Fourier series, bounding the size of the truncated tails. (Other manifolds or other dynamics could require other Banach spaces and other types of approximations.) In particular, the Fourier model we use to manage Fourier series rigorously is a trigonometric polynomial with interval coefficients plus an interval error. We emphasize that suitable Fourier and Lindstedt (Fourier-Taylor) models are ubiquitous in computer-assisted proofs in KAM theory and renormalization theory $[13,19,18,42,43,49]$. Our validation algorithms for proving the existence of invariant tori were implemented using our own $\mathrm{C}++$ library to manipulate Fourier models together with the rigorous interval library FILIB++ Interval Library; see [48]. All the validations presented here were tested with several types of computers working under several operating systems, although we report only the results obtained with a machine with Intel Core2 Quad CPU Q9550 at $2.83 \mathrm{GHz}$ working under Debian, using one of the processors.

1.4. Notation. $\mathbb{R}^{n}$ denotes the $n$-dimensional real space, and $e_{1}, \ldots e_{n}$ represents its unit vectors that form the standard basis. For $i=1, \ldots n$ and $v \in \mathbb{R}^{n}, \pi_{i} v$ is the $i$ th component of the vector $v . \mathrm{L}\left(\mathbb{R}^{n} ; \mathbb{R}^{k}\right)$ is the space of linear maps from $\mathbb{R}^{n}$ to $\mathbb{R}^{k}$, identified by the set of $k \times n$ matrices. The space of endomorphism of $\mathbb{R}^{n}$, identified by the set of square $n \times n$ matrices, is $\mathrm{L}\left(\mathbb{R}^{n}\right)=\mathrm{L}\left(\mathbb{R}^{n} ; \mathbb{R}^{n}\right)$, and $\mathrm{GL}\left(\mathbb{R}^{n}\right)$ is its subgroup of automorphisms, i.e., the group of invertible $n \times n$ matrices. $\mathrm{I}_{n}$ represents the $n \times n$ identity matrix. $\mathrm{L}\left(\mathbb{R}^{n}, \mathbb{R}^{m} ; \mathbb{R}^{k}\right)$ denotes the set of bilinear maps from $\mathbb{R}^{n} \times \mathbb{R}^{m}$ to $\mathbb{R}^{k}$. We also denote $\mathrm{L}^{2}\left(\mathbb{R}^{n} ; \mathbb{R}^{k}\right)=\mathrm{L}\left(\mathbb{R}^{n}, \mathbb{R}^{n} ; \mathbb{R}^{k}\right)$ and $\mathrm{L}^{2}\left(\mathbb{R}^{n}\right)=\mathrm{L}^{2}\left(\mathbb{R}^{n} ; \mathbb{R}^{n}\right)$. If we are given norms in $\mathbb{R}^{n}, \mathbb{R}^{m}, \mathbb{R}^{k}$, we consider the induced norms in the spaces of linear maps and bilinear maps previously mentioned. For instance, if we consider the maximum norm in $\mathbb{R}^{n}, \mathbb{R}^{m}, \mathbb{R}^{k}$ for $M \in \mathrm{L}\left(\mathbb{R}^{n} ; \mathbb{R}^{k}\right)$ and $B \in \mathrm{L}\left(\mathbb{R}^{n}, \mathbb{R}^{m} ; \mathbb{R}^{k}\right)$ we have $|M|=\max _{i} \sum_{j}\left|\pi_{i} M e_{j}\right|$ and $|B|=\max _{i} \sum_{r, s}\left|\pi_{i} B\left(e_{r}, e_{s}\right)\right|$.

$\mathbb{T}=\mathbb{R} / \mathbb{Z}$ denotes the one-dimensional torus. The translation on the torus of frequency $\omega \in \mathbb{R}$ is the map $t_{\omega}: \mathbb{T} \rightarrow \mathbb{T}$ defined as $t_{\omega}(\theta)=\theta+\omega$.

$\mathbb{R}^{n} \times \mathbb{T}$ denotes a trivial bundle over $\mathbb{T}$ (with projection $\pi: \mathbb{R}^{n} \times \mathbb{T} \rightarrow \mathbb{T}$ ). We assume that this bundle is endowed with a Finslered norm, i.e., a norm $|\cdot|_{\theta}$ on each fiber $\mathbb{R}^{n} \times\{\theta\}$ that depends continuously on $\theta$. We typically omit the subindex $\theta$ when the fiber is understood (or if the norm does not depend on $\theta$ ). A strip in the bundle is a set $D \subset \mathbb{R}^{n} \times \mathbb{T}$ such that

Copyright (c) by SIAM. Unauthorized reproduction of this article is prohibited. 
for $\pi(D)=\mathbb{T}$. For each $\theta \in \mathbb{T}, D_{\theta}=\left\{z \in \mathbb{R}^{n} \mid(z, \theta) \in D\right\} \subset \mathbb{R}^{n}$. A typical example is $D=U \times \mathbb{T}$, where $U \subset \mathbb{R}^{n}$ is open.

For a vector space $Z$ and a Finslered norm in the trivial bundle $Z \times \mathbb{T}$ over $\mathbb{T}$, we identify the space of continuous sections of the bundle with the set of continuous functions $\sigma: \mathbb{T} \rightarrow Z$, $C^{0}(\mathbb{T} ; Z)$, endowed with the supremum norm $\|\sigma\|=\sup _{\theta \in \mathbb{T}}|\sigma(\theta)|_{\theta}$.

2. A validation algorithm for robust invariant tori. In this section we review some definitions and results on fiberwise hyperbolic invariant tori (FHIT) for skew products over rotations. In particular, we state Theorem 1 in [27] for existence and local uniqueness of FHIT, which is the basis for our validation algorithms of existence and local uniqueness of FHIT.

2.1. FHIT. A (discrete) quasi-periodic system (with frequency $\omega \in \mathbb{R} / \mathbb{Q}$ ) is described by a skew product over a rotation

$$
\begin{aligned}
& \left(F, t_{\omega}\right): \quad D \subset \mathbb{R}^{n} \times \mathbb{T} \longrightarrow \mathbb{R}^{n} \times \mathbb{T}, \\
& (z, \theta) \quad \longrightarrow(F(z, \theta), \theta+\omega) \text {, }
\end{aligned}
$$

where $F: D \subset \mathbb{R}^{n} \times \mathbb{T} \rightarrow \mathbb{R}^{n}$ is continuous in the open strip $D$. Throughout this paper, we assume that $F$ is $C^{2}$ with respect to $z$. The bundle map (2.1) induces a graph transform functional $\mathcal{F}: C^{0}\left(\mathbb{T}, \mathbb{R}^{n}\right) \rightarrow C^{0}\left(\mathbb{T}, \mathbb{R}^{n}\right)$, defined as

$$
\mathcal{F}(K)(\theta)=F(K(\theta-\omega), \theta-\omega) .
$$

Notice that the graph of a continuous map $K: \mathbb{T} \rightarrow \mathbb{R}^{n}$ is a torus that is a copy of the base $\mathbb{T}$, and it is invariant under the skew product $\left(F, t_{\omega}\right)$ iff

$$
\mathcal{F}(K)=K \text {. }
$$

We will slightly abuse notation and refer to $K$ as a torus.

The linearized dynamics around a torus $K$ is given by the vector bundle map

$$
\begin{aligned}
\left(M_{K}, t_{\omega}\right): \begin{array}{l}
\mathbb{R}^{n} \times \mathbb{T} \\
(v, \theta) \longrightarrow
\end{array} \mathbb{R}^{n} \times \mathbb{T}, \\
\left(M_{K}(\theta) v, \theta+\omega\right),
\end{aligned}
$$

where $M_{K}: \mathbb{T} \rightarrow L\left(\mathbb{R}^{n}\right)$ is the transfer matrix $M_{K}(\theta)=\mathrm{D}_{z} F(K(\theta), \theta)$. We also refer to $\left(M_{K}, t_{\omega}\right)$ as the cocycle induced by $\left(F, t_{\omega}\right)$ and $K$. A cocycle $\left(M_{K}, t_{\omega}\right)$ induces a transfer operator $\mathcal{M}_{K}: C^{0}\left(\mathbb{T} ; \mathbb{R}^{n}\right) \rightarrow C^{0}\left(\mathbb{T} ; \mathbb{R}^{n}\right)$, defined as

$$
\mathcal{M}_{K}(V)(\theta)=M_{K}(\theta-\omega) V(\theta-\omega) .
$$

Notice that $\mathrm{D} \mathcal{F}(K)=\mathcal{M}_{K}$. We will suppress the dependence of $K$ when it is clear from the context.

The relation between the dynamical properties of cocycles and the spectral properties of the associated transfer operators has been intensively studied in the literature; see, e.g., [51, $58,34,50,47,16]$. These are important to describe the dynamical and functional properties of invariant objects and dynamical systems. We define now (both dynamically and functionally), the main geometric object of this paper.

Copyright $\odot$ by SIAM. Unauthorized reproduction of this article is prohibited. 
Definition 2.1. A FHIT of the system (2.1) is an invariant torus $K: \mathbb{T} \rightarrow \mathbb{R}^{n}$ such that its corresponding cocycle $\left(M_{K}, t_{\omega}\right)$ is uniformly hyperbolic, that is, there exists a continuous decomposition of the vector bundle $\mathbb{R}^{n} \times \mathbb{T}$ in a Whitney sum $\mathcal{S} \oplus \mathcal{U}$ of two invariant bundles $\mathcal{S}$ and $\mathcal{U}$, such that $M$ restricted to $\mathcal{U}$ is invertible, and there exist constants $C>0$ and $0<\lambda<1$ such that

(i) if $(v, \theta) \in \mathcal{S}$, then $|M(\theta+(\ell-1) \omega) \cdots M(\theta) v| \leq C \lambda^{\ell}|v|$ for all $\ell \geq 0$;

(ii) if $(v, \theta) \in \mathcal{U}$, then $\left|M(\theta+\ell \omega)^{-1} \cdots M(\theta-\omega)^{-1} v\right| \leq C \lambda^{-\ell}|v|$ for all $\ell \leq 0$.

Definition 2.2. A FHIT of the system (2.1) is an invariant torus $K: \mathbb{T} \rightarrow \mathbb{R}^{n}$ such that its corresponding transfer operator $\mathcal{M}_{K}$ is hyperbolic, i.e., its spectrum has empty intersection with the unit circle $\{\lambda \in \mathbb{C}:|\lambda|=1\}$.

It turns out that both definitions are equivalent. The stable bundle $\mathcal{S}$ and the unstable bundle $\mathcal{U}$ of a cocycle $\left(M_{K}, t_{\omega}\right)$ are constructed from the spectral projections associated to the transfer operator $\mathcal{M}_{K}$ (and the spectral gap in the unit circle). The width of the spectral gap is given by the hyperbolicity constant $\lambda$ that measures the hyperbolicity of the cocycle. The uniformity of the hyperbolicity property around the torus is given by the uniformity constant $C$, which is related to the norms of the spectral projections (and hence with the shapes of the bundles). If the spectrum of $\mathcal{M}_{K}$ is inside the unit circle, then we say that the torus $K$ is an attractor and $\mathcal{U}$ is the zero bundle. If the spectrum of $\mathcal{M}_{K}$ is outside the unit circle, then the torus $K$ is a repeller and $\mathcal{S}$ is the zero bundle. Otherwise we will say that the torus $K$ is a saddle.

Remark 2.3. We emphasize that there is a bootstrap in the regularity of a FHIT, and even though in Definition 2.1 we assume that it is continuous, in fact it is as smooth as the system [28].

Remark 2.4. Most of the above also works with slight modifications for invariant graphs of skew products over homeomorphisms. But fiberwise hyperbolic invariant graphs are in general less regular than the system.

Remark 2.5. Since $\omega$ is irrational, the spectrum of the transfer operator $\mathcal{M}$ is a set of annuli centered at 0 , and the inner annulus is a disk if the corresponding cocycle is noninvertible. This is also true in the generality of invariant graphs of skew products over homeomorphisms with a dense set of aperiodic points [51].

Remark 2.6. Transfer operators are bounded but noncompact operators. This fact makes the computation of their spectrum difficult [36].

In our methods of computation and validation of invariant tori, the crux is that a sufficient condition to apply Newton's method to solve the invariance equation

$$
\mathcal{F}(K)-K=0
$$

from an approximate solution $K_{0}$ is the invertibility of the bounded linear operator $\operatorname{DF}\left(K_{0}\right)-$ $\mathcal{I}=\mathcal{M}_{K_{0}}-\mathcal{I}$, and this is implied (in fact equivalent, since the rotation $\omega$ is irrational) by the hyperbolicity condition. Moreover, as a consequence of the implicit function theorem, FHIT are robust (persist) under perturbations of the system [28].

2.2. Reducibility. The standing hypothesis in our methodology is that the torus $K$ is fiberwise hyperbolic and the corresponding stable and unstable bundles are trivial (i.e., given by global frames). Hence, we can define a matrix valued map $P: \mathbb{T} \rightarrow G L\left(\mathbb{R}^{n}\right)$, whose first $n_{s}$

Copyright (c) by SIAM. Unauthorized reproduction of this article is prohibited. 
columns of $P, P^{s}$ parametrize the stable bundle $\mathcal{S}$ (of rank $n_{s}$ ) and the last $n_{u}$ columns of $P$, $P^{u}$ parametrize the unstable bundle $\mathcal{U}$ (of rank $n_{u}$ ). Since the bundles $\mathcal{S}$ and $\mathcal{U}$ are invariant, then

$$
P(\theta+\omega)^{-1} M_{K}(\theta) P(\theta)=\left(\begin{array}{cc}
\Lambda^{s}(\theta) & 0 \\
0 & \Lambda^{u}(\theta)
\end{array}\right),
$$

where $\Lambda^{s}: \mathbb{T} \rightarrow L\left(\mathbb{R}^{n_{s}}\right)$ and $\Lambda^{u}: \mathbb{T} \rightarrow G L\left(\mathbb{R}^{n_{u}}\right)$. In other words, a main assumption is that the cocycle $\left(M_{K}, t_{\omega}\right)$ is reducible to a block diagonal cocycle $\left(\Lambda, t_{\omega}\right)$, with $\Lambda=\left(\Lambda^{s}, \Lambda^{u}\right) . \Lambda^{s}$ and $\Lambda^{u}$ give the dynamics on the stable and unstable bundles, respectively.

Remark 2.7. Nontriviality of rank 1 bundles can easily be overcome with the double covering trick. See [29] for examples of computation of invariant tori with nonorientable bundles.

Using suitable adapted norms, one can bound the norms of the block diagonal cocycle obtaining $\max \left(\left\|\Lambda^{s}\right\|,\left\|\left(\Lambda^{u}\right)^{-1}\right\|\right) \leq \lambda$, with $\lambda<1$. Hence,

(i) if $(v, \theta) \in \mathcal{S}$, then $v=P^{s}(\theta) v^{s}$ with $v^{s} \in \mathbb{R}^{n_{s}}$ and $|M(\theta+(\ell-1) \omega) \cdots M(\theta) v| \leq$ $\|P\|\left\|P^{-1}\right\| \lambda^{\ell}|v|$ for all $\ell \geq 0$;

(ii) if $(v, \theta) \in \mathcal{U}$, then $v=P^{u}(\theta) v^{u}$ with $v^{u} \in \mathbb{R}^{n_{u}}$ and $\left|M(\theta+\ell \omega)^{-1} \cdots M(\theta-\omega)^{-1} v\right| \leq$ $\|P\|\left\|P^{-1}\right\| \lambda^{-\ell}|v|$ for all $\ell \leq 0$.

That is, the condition number $C=\|P\|\left\|P^{-1}\right\|$ of the adapted frame $P$ is the uniformity constant of the hyperbolic splitting.

An important situation in which linearized dynamics is very simple and estimates of the hyperbolicity constants can be, in principle, easily obtained is when the cocycle $\left(M_{K}, t_{\omega}\right)$ is reducible to a constant cocycle $\left(\Lambda_{0}, t_{\omega}\right)$ (possibly using the double covering trick). The linearized dynamics is then equivalent to iterating the constant matrix $\Lambda_{0}$, but the problem is obtaining the suitable adapted frame. We emphasize that invertible rank 1 cocycles are reducible to constants, under Diophantine conditions of the frequency $\omega$, while noninvertible rank 1 cocycles are not; see section 4. There are many other situations in which cocycles fail to be reducible (see, e.g., [33]), implying a complex behavior of the linearized dynamics $[29,26]$.

2.3. A validation theorem. In the previous subsection we grasped the relation between hyperbolicity and the applicability of Newton's method. From Theorem 1, p. 12, in [27], Newton's method for finding FHIT converges quadratically, provided that the initial approximations of the torus and its invariant bundles are fairly accurate. The following is a reformulation of such a theorem (see [27] for the proof), which is the theoretical core of the validations done in this paper.

Theorem 2.8. Let $\mathbb{R}^{n} \times \mathbb{T}$ be the Finslered trivial bundle over $\mathbb{T}$. Let $F: D \subset \mathbb{R}^{n} \times \mathbb{T} \rightarrow \mathbb{R}^{n}$ be a continuous map defined in an open strip $D, C^{2}$ with respect to $z$, and $\omega \in \mathbb{R}$, defining the skew product $\left(F, t_{\omega}\right): D \rightarrow \mathbb{R}^{n} \times \mathbb{T}$.

Assume we are given

(1.1) a continuous map $K: \mathbb{T} \rightarrow \mathbb{R}^{n}$, parameterizing a torus;

(1.2) two continuous matrix-valued maps $P_{1}, P_{2}: \mathbb{T} \rightarrow \mathrm{L}\left(\mathbb{R}^{n}\right)$, giving adapted frames;

(1.3) a continuous block diagonal matrix-valued map $\Lambda=\operatorname{diag}\left(\Lambda^{s}, \Lambda^{u}\right): \mathbb{T} \rightarrow \mathrm{L}\left(\mathbb{R}^{n}\right)$, where $\Lambda^{s}: \mathbb{T} \rightarrow \mathrm{L}\left(\mathbb{R}^{n_{s}}\right)$ and $\Lambda^{u}: \mathbb{T} \rightarrow \mathrm{GL}\left(\mathbb{R}^{n_{u}}\right)$ with $n=n_{s}+n_{u} ;$ 
(1.4) a closed strip $\bar{D}_{P_{1}}(K, r)=\left\{\left(K(\theta)+P_{1}(\theta) v, \theta\right)|\theta \in \mathbb{T}| v \mid, \leq r\right\} \subset D$, where $r>0$;

(1.5) a positive constant $b$ such that $B(z, \theta)=P_{2}(\theta+\omega) \mathrm{D}_{z}^{2} F(z, \theta)\left[P_{1}(\theta) \cdot, P_{1}(\theta) \cdot\right] \in L^{2}\left(\mathbb{R}^{n}\right)$ satisfies $|B(z, \theta)| \leq b$ for any $(z, \theta) \in \bar{D}_{P_{1}}(K, r)$.

Let $\sigma, \tau, \lambda$ be positive constants such that

(2.1) for each $\theta \in \mathbb{T}, T(\theta)=P_{2}(\theta) P_{1}(\theta)-\mathrm{I}_{n} \in \mathrm{L}\left(\mathbb{R}^{n}\right)$ satisfies $|T(\theta)| \leq \tau$;

(2.2) for each $\theta \in \mathbb{T}, S(\theta)=P_{2}(\theta+\omega) \mathrm{D} F(K(\theta), \theta) P_{1}(\theta)-\Lambda(\theta) \in \mathrm{L}\left(\mathbb{R}^{n}\right)$ satisfies $|S(\theta)| \leq \sigma$;

(2.3) for each $\theta \in \mathbb{T}, \max \left(\left|\Lambda^{s}(\theta)\right|,\left|\Lambda^{u}(\theta)^{-1}\right|\right) \leq \lambda$;

and assume that

(2.4) $\lambda+\sigma+\tau<1$.

Let $\rho, h$ be positive constants such that

(3.1) for each $\theta \in \mathbb{T}, R(\theta)=P_{2}(\theta+\omega)(F(K(\theta), \theta)-K(\theta+\omega)) \in \mathbb{R}^{n}$ satisfies $|R(\theta)| \leq \rho$;

(3.2) $(1-\lambda-\sigma-\tau)^{-2} b \rho \leq h$;

and assume that

(3.3) $h<\frac{1}{2}$.

Let $r_{0}, r_{1}$ be positive constants such that

(4.1) $(1-\lambda-\sigma-\tau)(1-\sqrt{1-2 h}) b^{-1} \leq r_{0}$;

(4.2) $r_{1} \leq \min \left((1-\lambda-\sigma-\tau)(1+\sqrt{1-2 h}) b^{-1}, r\right)$;

and assume that

(4.3) $r_{0} \leq r$.

Then, there exists a unique continuous map $K_{*}: \mathbb{T} \rightarrow \mathbb{R}^{n}$ such that

(a.1) for each $\theta \in \mathbb{T}, F\left(K_{*}(\theta), \theta\right)-K_{*}(\theta+\omega)=0$;

(a.2) for each $\theta \in \mathbb{T},\left|P_{1}^{-1}(\theta)\left(K_{*}(\theta)-K(\theta)\right)\right| \leq r_{1}$.

Moreover,

(a.3) for each $\theta \in \mathbb{T},\left|P_{1}^{-1}(\theta)\left(K_{*}(\theta)-K(\theta)\right)\right| \leq r_{0}$.

Let $\hat{\lambda}, \mu$ be positive constants such that

(5.1) for each $\theta \in \mathbb{T}, \max \left(\left|\Lambda^{s}(\theta)\right|,\left|\Lambda^{u}(\theta)\right|\right) \leq \hat{\lambda}$;

(5.2) $\lambda\left(1-\lambda^{2}\right)^{-1}(1-\tau)^{-1}\left(b r_{0}+\sigma+\hat{\lambda} \tau\right) \leq \mu$;

and assume that

(5.3) $\mu<\frac{1}{4}$.

Then, there exist a continuous matrix-valued map $P_{*}: \mathbb{T} \rightarrow \mathrm{GL}\left(\mathbb{R}^{n}\right)$ and a continuous blockdiagonal matrix-valued map $\Lambda_{*}=\operatorname{diag}\left(\Lambda_{*}^{s}, \Lambda_{*}^{u}\right): \mathbb{T} \rightarrow \mathrm{L}\left(\mathbb{R}^{n}\right)$, where $\Lambda_{*}^{s}: \mathbb{T} \rightarrow \mathrm{L}\left(\mathbb{R}^{n_{s}}\right)$ and $\Lambda_{*}^{u}: \mathbb{T} \rightarrow \mathrm{GL}\left(\mathbb{R}^{n_{u}}\right)$, such that

(b.1) for each $\theta \in \mathbb{T}, P_{*}(\theta+\omega)^{-1} \mathrm{D}_{z} F\left(K_{*}(\theta), \theta\right) P_{*}(\theta)-\Lambda_{*}(\theta)=0$;

(b.2) for each $\theta \in \mathbb{T},\left|P_{1}(\theta)^{-1}\left(P_{*}(\theta)-P_{1}(\theta)\right)\right| \leq r_{P}$, where $r_{P} \geq \frac{\mu}{\sqrt{1-4 \mu}}$;

(b.3) for each $\theta \in \mathbb{T},\left|\Lambda_{*}(\theta)-\Lambda(\theta)\right| \leq r_{\Lambda}$, where $r_{\Lambda} \geq\left(1+r_{P}\right)(1-\tau)^{-1}\left(b r_{0}+\sigma+\hat{\lambda} \tau\right)$.

The main idea behind Theorem 2.8 is to consider an adapted frame, given by the matrixvalued map $P_{1}$, in which the hyperbolicity properties are checked. This frame $P_{1}$ encodes the approximations of the stable and unstable bundles. In more detail, the conditions to be checked in Theorem 2.8 state the following:

(1) From the initial data, $K, P_{1}$ (and $P_{2}$ ) one constructs an adapted system of coordinates $(v, \theta)$ on a neighborhood of the torus, a strip $\bar{D}_{P_{1}}(K, r)$ :

$$
(v, \theta) \in\{(v, \theta)|\theta \in \mathbb{T},| v \mid \leq 1\} \rightarrow\left(z=K(\theta)+P_{1}(\theta) v, \theta\right) \in \bar{D}_{P_{1}}(K, r) .
$$

Copyright (C) by SIAM. Unauthorized reproduction of this article is prohibited. 
In these coordinates, and in this strip, the norm of the second differential of $F$ is bounded by $b$. Cocycle $\left(\mathrm{D} F(K(\theta), \theta), t_{\omega}\right)$ is approximately reducible (via $P_{1}$ and $P_{2}$ ) to the block diagonal cocycle $\left(\Lambda(\theta), t_{\omega}\right)$.

(2) Point (2.4) is the verification of the hyperbolicity property (and of the invertibility of $P_{1}$ ). This depends on the error bound in the approximate inverse $P_{2}$, the quality of the conjugacy to the block diagonal cocycle $\left(\Lambda, t_{\omega}\right)$, and the hyperbolicity property viewed in the adapted frame.

(3) The approximate invariance of the torus is estimated by $\rho$ in (3.1). If $\rho$ is small enough, then there will be a true invariant torus nearby. The first step is checking (3.3).

(4) Points (3.3) and (4.3) check the Newton-Kantorovich hypothesis for the validation of the existence and local uniqueness (in $\bar{D}_{P_{1}}\left(K, r_{1}\right)$ ) of a FHIT $K_{*}$ nearby $K$. An upper bound of the distance between $K_{*}$ and $K$, in the $P_{1}$ adapted frame, is given by $r_{0}$.

(5) Checking point (5.3) leads to a validation of the invariant bundles codified in $P_{*}$, providing rigorous upper bounds of the distance between the adapted frames $P_{1}$ and $P_{*}$ (and hence, between the approximate and true invariant bundles) and estimates of the hyperbolicity properties.

Remark 2.9. One can perform the bounds of Theorem 2.8 in the original coordinates, that is, compute $\rho_{0}$ and $b_{0}$ such that

$\left(1.5^{\prime}\right)$ for each $(z, \theta) \in D,\left|\mathrm{D}_{z}^{2} F(z, \theta)\right| \leq b_{0}$;

$\left(3.1^{\prime}\right)$ for each $\theta \in \mathbb{T},|F(K(\theta), \theta)-K(\theta+\omega)| \leq \rho_{0}$.

These estimates lead to (crude) bounds of $\rho$ and $b$. In particular, inequality (3.2) in Theorem 2.8 is rephrased as

$\left(3.2^{\prime}\right)(1-\lambda-\sigma-\tau)^{-2} C^{2} b_{0} \rho_{0} \leq h$,

where $C=\left\|P_{2}\right\|\left\|P_{1}\right\|$ is the condition number of the adapted frame. Notice that $h$ grows with the square of the hyperbolicity constant $C /(1-\lambda)$. Hence, the weaker the hyperbolic properties, the much harder it is to pass the Newton-Kantorovich test.

Remark 2.10. Theorem 2.8 is stated using $C^{0}$ norms. One can state a similar theorem using norms with higher regularities (e.g., $C^{r}$, Sobolev, analytic). In this paper we have only considered (and implemented) validations using $C^{0}$ norms. Hence, although the FHIT $K_{*}$ is as smooth as the skew product and the bundles are as smooth as its differential, we only measure the distance of the invariant objects to the approximately invariant objects using $C^{0}$ norms. We plan to come back to this problem in the future.

Remark 2.11. Theorem 2.8 works, with minor changes, if $\mathbb{T}$ is replaced by a general compact metric space and $t_{\omega}: \mathbb{T} \rightarrow \mathbb{T}$ is replaced by any homeomorphism.

3. Implementation of the validation algorithm. In this section we explain implementation issues of computer validations of FHIT in skew products over rotations, based on Theorem 2.8. Since the base manifold of the skew product is a torus, and the base dynamics is a rotation, we use Fourier polynomials to approximate the periodic functions to model the components of the approximate invariant tori and bundles of the input data for the algorithm (and this is the reason for assuming triviality of the bundles).

Theorem 2.8 assumes a Finslered norm. In our present implementation, we have considered the sup norm on each fiber. Hence, instead of considering adapted (Finslered) norms, we consider suitable adapted frames.

Copyright (c) by SIAM. Unauthorized reproduction of this article is prohibited. 
The core of the implementation is a set of routines to rigorously manage periodic functions and enclose them in Fourier polynomials plus error intervals. These are what we refer to as the Fourier models and are briefly introduced in subsection 3.1 and in Appendix A.

The validating computer program has to verify, from an approximately invariant torus and approximately invariant stable and unstable bundles (e.g., computed numerically or using perturbative arguments), all the hypotheses of Theorem 2.8. Notice that the checking has to be done only once. Since we will apply the computer programs in situations in which tori are about to break (see sections 4 and 5), we prioritize the accuracy over the speed of the computations.

3.1. Fourier models. Here we detail the definition of Fourier models, assuming the reader is familiar with interval computations $[52,62,64]$. In what follows, when we refer to interval we mean a compact interval. Given a (compact) interval $J=[a, b]$. The modulus of an interval is $|J|=\max (|a|,|b|)$. Following the standard findings in the literature, the result of an operation with intervals is an interval that encloses the result. This is what one can do when implementing interval operations in a computer.

Definition 3.1. A (one-dimensional, real) Fourier model of order $m \geq 0$ is a couple $\hat{G}=$ $(G(\theta), R)$, where

$$
G(\theta)=A_{0}+\sum_{k=1}^{m}\left(A_{k} \cos (2 \pi k \theta)+B_{k} \sin (2 \pi k \theta)\right)
$$

is a trigonometric polynomial with interval coefficients $A_{0}, \ldots, A_{m}, B_{1}, \ldots B_{m}$, and the remainder $R=R(\hat{G})$ is an interval. Abusing notation, we denote $B_{0}=\{0\}$, and for all $k>m$ we define $A_{k}=B_{k}=\{0\}$.

We say that a continuous function $g: \mathbb{T} \rightarrow \mathbb{R}$ belongs to the Fourier model $\hat{G}$, denoted $g \in \hat{G}$, if for all $\theta \in \mathbb{T}, g(\theta) \in A_{0}+\sum_{k=1}^{m}\left(A_{k} \cos (2 \pi k \theta)+B_{k} \sin (2 \pi k \theta)\right)+R$.

Let $\hat{G}, \hat{H}$ be two Fourier models. We say that $\hat{G}$ is enclosed by $\hat{H}, \hat{G} \subset \hat{H}$, iff for any continuous periodic function $g: \mathbb{T} \rightarrow \mathbb{R}, g \in \hat{G}$ implies $g \in \hat{H}$.

Given an interval $J$, the image of $J$ under the Fourier model $\hat{G}$ is defined as $\hat{G}(J)=$ $G(J)+R(\hat{G})$, where $G(J)$ is the interval image of $J$ under the trigonometric polynomial with interval coefficients $G$. That is, $\hat{G}(J)=\{f(\theta) \in \mathbb{R} \mid f \in \hat{G}, \theta \in J\}$. The image of $\hat{G}$ is $\hat{G}([0,1])$. The supremum norm is the nonnegative number $\|\hat{G}\|=|\hat{G}([0,1])|$. Upper bounds of the supremum norm are $\left|A_{0}\right|+\sum_{k=1}^{m}\left(\left|A_{k}\right|+\left|B_{k}\right|\right)+|R|$ and $\left|A_{0}\right|+\sum_{k=1}^{m} \sqrt{\left|A_{k}\right|^{2}+\left|B_{k}\right|^{2}}+|R|$.

Remark 3.2. The computer implementation of $\hat{G}(J)$ obtains an enclosure $E$ of the result, i.e., $\hat{G}(J) \subset E$. In order to avoid large overestimations, especially in cases in which the functions $f \in \hat{G}$ behave wildly, we subdivide the interval $J$ and compute the enclosures of the subdivisions.

Given a $d$-variable function $\varphi\left(x_{1}, \ldots, x_{d}\right)$ and $d$ Fourier models $\hat{G}_{1}, \ldots, \hat{G}_{d}$, we are interested in computing a Fourier model $\hat{H}$ enclosing the composition $\varphi \circ \hat{G}$, where $\hat{G}=$ $\left(\hat{G}_{1}, \ldots, \hat{G}_{d}\right)$. That is, we want that for any continuous functions $g_{1} \in \hat{G}_{1}, \ldots, g_{d} \in \hat{G}_{d}$, $\varphi \circ g \in \hat{H}$, where $g=\left(g_{1}, \ldots, g_{d}\right)$. Appendix A explains some algorithms to compute enclosures of compositions of Fourier models with some elementary functions.

3.2. Validation of FHIT. Here we show how Theorem 2.8 can be implemented, via Fourier models, in order to validate some initial data as a good approximation of a FHIT and its

Copyright (c) by SIAM. Unauthorized reproduction of this article is prohibited. 
invariant subbundles for a given continuous skew product $\left(F, t_{\omega}\right): D \subset \mathbb{R}^{n} \times \mathbb{T} \rightarrow \mathbb{R}^{n} \times \mathbb{T}, C^{2}$ with respect to $z$. The Finslered norm we consider in $\mathbb{R}^{n} \times \mathbb{T}$ is the sup norm on each fiber.

We assume that we can effectively compute the enclosures of the components of the compositions of $F(z, \theta), \mathrm{D}_{z} F(z, \theta)$ and $\mathrm{D}_{z}^{2} F(z, \theta)$ with Fourier models. That is, we can substitute $z$ by a (vector) Fourier model $\hat{K}$ if for each $\theta \in[0,1], \hat{K}(\theta) \subset D_{\theta}$ (a fact that can be rigorously checked by using interval arithmetic).

The input data of the validation algorithm follows:

(0) Compute, e.g., numerically or using perturbative arguments, the trigonometric polynomial approximations of an invariant torus $(K)$, the adapted frame $\left(P_{1}\right)$ and its inverse $\left(P_{2}\right)$, and the dynamics on the invariant bundles $\left(\Lambda=\operatorname{diag}\left(\Lambda^{s}, \Lambda^{u}\right)\right)$. References $[27$, $29]$ include algorithms and numerical computations of invariant tori and their bundles. The order $m$ of the approximations depends on the decay of the coefficients of the Fourier expansions (and hence on the quality of the initial data). We take $m$ in such a way that the size of the discarded term is below a given threshold (say, $10^{-6}$ ).

The validation algorithm mimics the statement of Theorem 2.8. Here are the steps:

(1) From the input data, derive the Fourier models $\hat{K}, \hat{P}_{1}, \hat{P}_{2}, \hat{\Lambda}=\left(\hat{\Lambda}^{s}, \hat{\Lambda}^{u}\right)$.

Introduce a radius $r$, and compute a Fourier model $\hat{K}_{r}=K+P_{1}[-r, r]^{n} \in \bar{D}_{\theta}$. Check (using interval aritmetics) that for all $\theta \in \mathbb{T}, \hat{K}_{r}(\theta) \in D_{\theta}$. If not, the algorithm stops. Then, compute the Fourier model $\hat{B}=B\left(\hat{K}+\hat{P}_{1}[-r, r]^{n}, \cdot\right)$ of the bilinear map $B(z, \theta)$ for points $(z, \theta)$ in the strip $\bar{D}_{P_{1}}(K, r)$. Compute an upper bound of the maximum norm of $\hat{B}, b$.

(2) Compute the upper bounds $\sigma, \tau, \lambda$ by enclosing the Fourier models $\hat{S}, \hat{T}, \hat{\Lambda}^{s},\left(\hat{\Lambda}^{u}\right)^{-1}$. Check (using interval arithmetic) if $\lambda+\sigma+\tau<1$. If not, hyperbolicty is not validated, and the algorithm stops.

(3) Compute the upper bounds $\rho$ of the error in the invariance equation from the Fourier model $\hat{R}$. Then, compute an upper bound $h$ of $(1-\lambda-\sigma-\tau)^{-2} b \rho$, and check if $h<\frac{1}{2}$. If not, the torus is not validated and the algorithm stops.

(4) Compute an upper bound $r_{0}$ of $(1-\lambda-\sigma-\tau)(1-\sqrt{1-2 h}) b^{-1}$, and check if $r_{0} \leq r$. If not, the torus is not validated and the algorithm stops.

After validating these steps, the torus is validated, meaning that there is a unique invariant torus $K_{*}$ in the strip $\bar{D}_{P_{1}}\left(K, r_{1}\right)$, where $r_{1}$ is a lower bound of $(1-\lambda-\sigma-\tau)(1+\sqrt{1-2 h}) b^{-1}$ and $r$. Moreover, the torus $K_{*}$ is contained in the strip $\bar{D}_{P_{1}}\left(K, r_{0}\right)$.

Remark 3.3. Bound $b$ (and subsequently $h$ ) depends on the radius $r$ of the strip. This choice has consequences on the estimates of the error radius $r_{0}$, which should be not greater than $r$, and the uniqueness radius $r_{1}$. In our actual implementation, the choice is taking $2(1-\lambda-\sigma-\tau)^{-1} \rho \leq r$ (hence $r$ is not given, but computed!), which ensures (if $h<\frac{1}{2}$ ) that $r_{0} \leq r$. By tuning $r$ one can improve $r_{0}$ and $r_{1}$.

The final step of the validation algorithm is checking the invariance of the adapted frame, as follows:

(5) Compute the upper bound of $\mu$ using $\sigma, \tau, \lambda, \hat{\lambda}, b, r_{0}$. Check if $\mu<\frac{1}{4}$. If not, the adapted frame is not validated, and the algorithm stops.

Else, there is an invariant frame $P_{*}$, codifying the stable and unstable bundles of $K_{*}$. The upper bounds $r_{P}$ and $r_{\Lambda}$ are rigorous estimates of the distances between the approximate and true adapted frame and hyperbolic dynamics, respectively.

Copyright $\odot$ by SIAM. Unauthorized reproduction of this article is prohibited. 
3.3. Validation of a family of FHIT. Here we show the procedure to validate the existence of a family of FHIT of a one-parameter family of skew products $\left(F_{s}, t_{\omega}\right): D \subset \mathbb{R}^{n} \times \mathbb{T} \rightarrow \mathbb{R}^{n} \times \mathbb{T}$ with parameter $s \in[a, b]$.

Consider the interval $[a, b]=I \cup J$, where $I$ and $J$ are closed intervals, and let $K_{i}, P_{1, i}$, $P_{2, i}$, and $\Lambda_{i}$ for $i=I, J$ be the initial data of the validation algorithm for the (interval) skew products $\left(F_{i}, t_{\omega}\right)$. In order to check that the corresponding validated tori belong to the same family we proceed as follows:

(0) Apply the validation algorithm explained in subsection 3.2 to the (interval) skew products $\left(F_{i}, t_{\omega}\right), i=I, J$. Besides the Fourier models corresponding to the initial data, $\hat{K}_{i}$, $\hat{P}_{1, i}, \hat{P}_{2, i}$, and $\hat{\Lambda}_{i}$, the validation algorithm produces bounds $\rho_{i}, \sigma_{i}, \tau_{i}, r_{0, i}, r_{1, i}, h_{i}$.

(1) Construct the Fourier model $\hat{E}_{I, J}=\hat{P}_{2, J} \cdot\left(\hat{K}_{J}-\left(\hat{K}_{I}+\hat{P}_{1, I} \cdot\left[-r_{0, I}, r_{0, I}\right]^{n}\right)\right)$. Check if

$$
\left\|\hat{E}_{I, J}\right\| \leq\left(1-\tau_{J}\right) r_{1, J}
$$

If this holds, the two initial data approximate the same family of FHIT; if not, this family has not been validated.

4. Example 1: Computer validations for noninvertible skew products. In this section we report computer validations of existence of invariant tori for a noninvertible map, the quasiperiodically driven logistic map (1.1), proving Proposition 1.1. Special emphasis is placed on validation of nonreducible tori for values close to their breakdown. Note that in this context, the concept of nonreducible torus is equivalent to the noninvertibility of the transfer matrix along the torus.

Recall from (1.1) that the quasi-periodically driven logistic map is the skew product

$$
\begin{aligned}
\left(F, t_{\omega}\right): & \mathbb{R} \times \mathbb{T} \\
(z, \theta) & \longrightarrow \mathbb{R} \times \mathbb{T}, \\
&
\end{aligned}
$$

where here we fix $\omega=\frac{1}{2}(\sqrt{5}-1)$ and $a$ and $D$ are parameters. In this section, we fix $D=0.1$ and let $a>0$ vary. The critical curve, where derivative of $F$ with respect to $z$ vanishes, is $\mathcal{C}=\left\{z=\frac{1}{2}\right\}$.

We start with a numerical exploration of the model, then we face the computational problems produced by noninvertibility and deterioration of uniform hyperbolicity, and, after careful numerical computations of the input data for the validation algorithm, we apply such an algorithm to validate the existence of period 2 attracting tori for parameter values very close to the threshold.

4.1. Numerical exploration. Figure 3(a) shows the bifurcation diagram of the invariant objects, while Figure 3(b) shows the corresponding Lyapunov multipliers. A particularly simple case is the zero-curve $x_{a}(\theta)=0$, for which the Lyapunov multiplier can be analytically computed (see, e.g., [37]): $\Lambda_{0}(a)=\frac{a}{2}\left(1+\sqrt{1-D^{2}}\right)$. Hence, for $D=0.1$, the zero-curve $K_{0}$ is attracting if $a<a_{\mathrm{t}}$ and repelling if $a>a_{\mathrm{t}}$, and in $a_{\mathrm{t}}=2(1+\sqrt{0.99})^{-1}$ there is a transcritical bifurcation. Now, let's explain the other invariant curves and their bifurcations, labeled in Figure 3(b). This is done through a numerical exploration.

Copyright $\odot$ by SIAM. Unauthorized reproduction of this article is prohibited. 


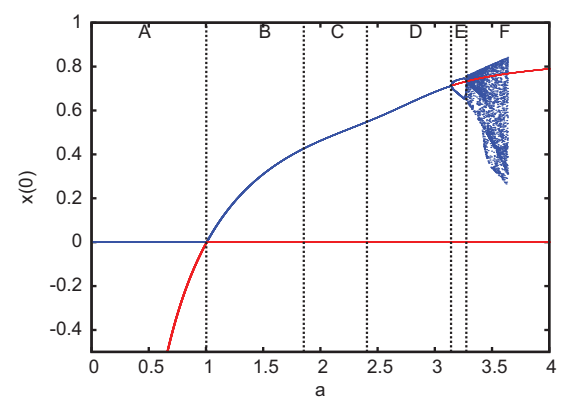

(a) $x(0)$ value of the invariant curves $x(\theta)$ with respect to parameter $a$.

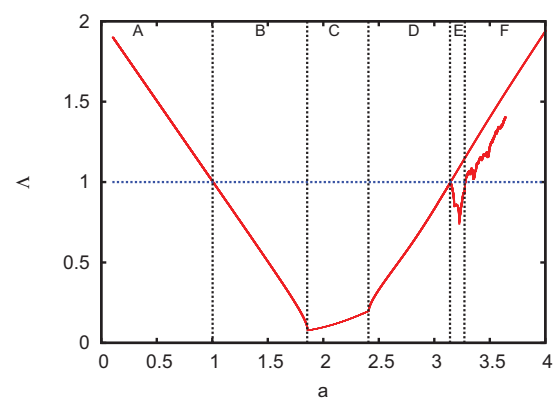

(b) The Lyapunov multiplier of the invariant curves.

Figure 3. Bifurcation diagram of the invariant curves and their Lyapunov multipliers, with respect to parameter $a$. Red represents a repelling curve and blue an attracting object. See text for details.

(A) $a \in\left(0, a_{\mathrm{t}}\right)$. There is a reducible repelling curve $K_{1}(a)$, and its cocycle is reducible to a constat $\left.\Lambda_{1}(a) \in\right] 1,+\infty$ [. In fact, as $a \rightarrow 0, K_{1}(a) \sim \frac{a-1}{a}$ and hence goes to $-\infty$, and $\Lambda_{1}(a) \rightarrow 2$. As $a \rightarrow a_{\mathrm{t}}$ this curve tends (uniformly) to the zero curve $K_{0}$, and $\Lambda_{1}(a) \rightarrow 1$.

At $a=a_{\mathrm{t}}=2(1+\sqrt{0.99})^{-1}$ there is a transcritical bifurcation (with $K_{0}$ ).

(B) $a \in\left(a_{\mathrm{t}}, a_{\mathrm{r}, 1}\right) . K_{1}(a)$ is a reducible attracting curve, and its cocycle is reducible to a positive constant $\left.\Lambda_{1}(a) \in\right] 0,1[$.

At $a=a_{\mathrm{r}, 1} \simeq 1.854419$, the curve $K_{1}(a)$ is tangent to the critical curve $\mathcal{C}$.

(C) $a \in\left(a_{\mathrm{r}, 1}, a_{\mathrm{r}, 2}\right) . K_{1}(a)$ is a nonreducible attracting curve, since its transfer matrix vanishes at the points in which $K_{1}(a)$ intersects the critical curve $\mathcal{C}$.

At $a=a_{\mathrm{r}, 2} \simeq 2.406952$, the curve $K_{1}(a)$ is again tangent to the critical curve $\mathcal{C}$.

(D) $a \in\left(a_{\mathrm{r}, 2}, a_{\mathrm{p}}\right)$. The curve $K_{1}(a)$ is a reducible attracting curve, and its cocycle is reducible to a negative constant $\left.\Lambda_{1}(a) \in\right]-1,0[$.

At $a_{\mathrm{p}} \simeq 3.141875, \Lambda_{1}(a)=-1$, and $K_{1}(a)$ has a period doubling bifurcation, in which a period 2 attracting curve $K_{2}(a)$ is born.

(E) $a \in\left(a_{\mathrm{p}}, a_{\mathrm{c}}\right) \cdot K_{1}(a)$ is a reducible repelling curve, and its cocycle is reducible to a negative constant $\left.\Lambda_{1}(a) \in\right]-\infty,-1\left[\right.$. There is also a period 2 attracting curve $K_{2}(a)$ (see Figures 4(a) and 4(b) for the corresponding Lyapunov multipliers).

At $a=a_{\mathrm{c}} \simeq 3.271383$, the period 2 attracting curve collides in a nonsmooth way with the repelling curve, bifurcating in an SNA.

(F) $a \in\left[a_{\mathrm{c}}, \infty\right)$. The reducible repelling curve $K_{1}$ survives after the collision and it exists for all these values. There is also a strange attractor, a geometrically complex attracting object that comes from the destruction of $K_{2}$.

We have focused our study in region (E), very far from the perturbative regime. This is known as the Heagy-Hammel fractalization route to SNA. Figures 5(a) and 5(b) show these invariant objects before and after the collision at $a=a_{\mathrm{c}}$. Remarkably, the main ingredient in this route is the loss of reducibility that the period 2 attracting curve suffers at $a=a_{\mathrm{r}} \simeq$ 3.17496 , related to the nonsmooth collision with the repelling curve at $a=a_{\mathrm{c}} \simeq 3.271383$.

We have validated both the repelling curve and the period 2 attracting curve in region E. Of course, our techniques can also be applied to analyzing cases (A), (B), (C), and (D). 


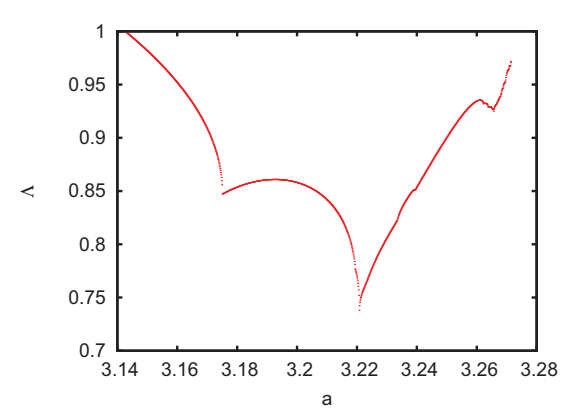

(a) Lyapunov multiplier of the period 2 attracting curve in region $(\mathrm{E})$. The peaks correspond to variations of the number of zeroes of the transfer cocycle [37].

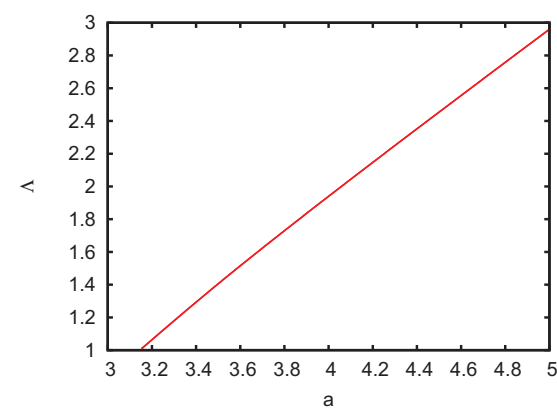

(b) Lyapunov multiplier of the repelling curve in regions $(\mathrm{E})$ and $(\mathrm{F})$. There is no trace of the nonsmooth (E)-(F) bifurcation of the period 2 attracting companion around $a=3.271$.

Figure 4. Lyapunov multipliers of the invariant and periodic curves with respect to parameter a.

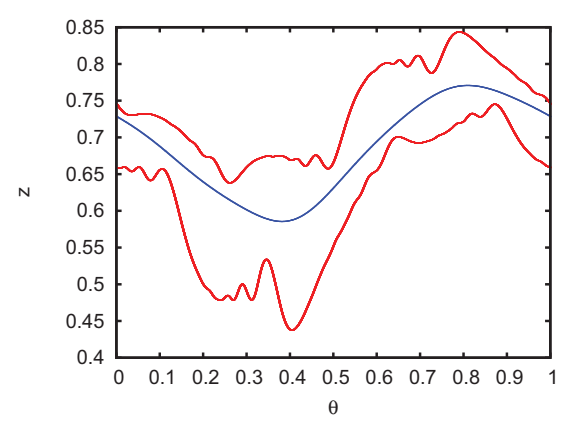

(a) Invariant curves for $a=3.24$. The red curve is the period 2 attractor, and the blue curve is the repellor.

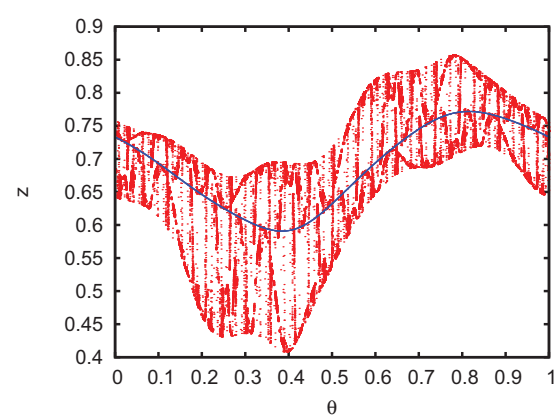

(b) Invariant objects for $a=3.272$. The red object is the SNA, and the blue curve is the repellor.

Figure 5. Graphical representation of the Heagy-Hammel route. See text for details.

In the smooth bifurcations at $a=a_{\mathrm{t}}($ in $(\mathrm{A})-(\mathrm{B}))$ and $a=a_{\mathrm{p}}$ (in (D)-(E)) the invariant tori are reducible. The attracting torus in region $(\mathrm{C})$ is not reducible, but it is far from destruction. Hence, the study in region (E) close to $a=a_{\mathrm{p}}$ is an example of system close to smooth (reducible) bifurcation and close to $a=a_{\mathrm{c}}$ is an example of system close to nonsmooth (nonreducible) bifurcation and can be applied to the other cases.

Remark 4.1. Nonreducibility region (C) separates region (B), in which the torus is attracting with a well-defined positive eigenvalue, and region (D), in which the torus is attracting with a well-defined negative eigenvalue. In region (C) we cannot define an eigenvalue, since the cocycle is not reducible to constant. But we can define a Lyapunov multiplier (which in reducible cases is the absolute value of the eigenvalue), which in the region (C) does not cross 0 . Hence, the sign of the eigenvalue jumps (from + to - ) in region $(\mathrm{C})$. Interestingly, this phenomenon of jump of the sign has been observed in other quasi-periodic systems [29, 26].

4.2. Numerical computation of the initial data. In this section, we describe how to compute the initial data $K, P_{1}, P_{2}, \Lambda$ for attracting curves of the noninvertible one-dimensional 
skew product $\left(F, t_{\omega}\right)$. Similar methods can be applied for repelling curves by using a right inverse of the map (i.e., one of the branches of the inverse of $\left.\left(F, t_{\omega}\right)\right)$. We will also present methods to deal with noninvertible transfer matrices.

The approximately invariant torus $K$ can be computed using the simple iteration algorithm, since the invariant torus is attracting. The number of iterations needed to obtain a good approximation depends heavily on the modulus of the Lyapunov multiplier. In our computations, the number of iterations does not exceed $10^{10}$.

More challenging is the computation of the initial data $P_{1}, P_{2}, \Lambda$, since even though the transfer matrix $M$ is contracting "on average," it can be locally expanding. The condition of invertibility of the transfer matrix plays a key role in this computation. We have considered two methods in order to overcome these computational problems.

Lyapunov metric. This is a general construction when dealing with uniform hyperbolicity [3] that we adapt for the one-dimensional case. For a uniformly attracting torus with transfer matrix $M$ and Lyapunov multiplier $\lambda$, this metric is given by $|v|_{\theta}=|L(\theta) v|$, where $L: \mathbb{T} \rightarrow$ $[1, \infty)$ is the continuous function

$$
L(\theta)=\sum_{j=0}^{\infty} \frac{1}{\bar{\lambda}^{j}}|M(\theta+(j-1) \omega) \cdots M(\theta)|,
$$

where $1>\bar{\lambda}>|\lambda|+\varepsilon$, for sufficiently small $\varepsilon>0$. Instead of considering this Lyapunov metric, we consider the transformations $P_{1}(\theta)=\frac{1}{L(\theta)}$ and $P_{2}(\theta)=L(\theta)$. Hence, we define the continuous function

$$
\Lambda(\theta)=P_{2}(\theta+\omega) M(\theta) P_{1}(\theta)=\operatorname{sgn}(M(\theta))\left(\frac{L(\theta)-1}{L(\theta)}\right) \bar{\lambda},
$$

where $\operatorname{sgn}(\cdot)$ is the sign function. Then, $|\Lambda(\theta)| \leq \bar{\lambda}<1$ for all $\theta \in \mathbb{T}$.

Reducibility and almost reducibility to constant coefficients. The goal of the reducibility method is to reduce the transfer matrix to a constant $\Lambda$, which satisfies

$$
M(\theta) P_{1}(\theta)=P_{1}(\theta+\omega) \Lambda,
$$

for a suitable transformation $P_{1}(\theta) \neq 0$. If $M(\theta)$ is invertible for all $\theta \in \mathbb{T}$, this equation is solved by taking logarithms and solving the small divisor equations obtained by matching the Fourier coefficients.

If $M(\theta)$ has zeroes, (4.4) has no continuous solutions. Hence, we cannot reduce $M(\theta)$ to constant coefficients. To overcome this difficulty, we consider the modified equation

$$
\left(M(\theta)^{2}+\varepsilon \eta(\theta)\right) P_{1}(\theta)^{2}=P_{1}(\theta+\omega)^{2} \lambda_{\varepsilon}^{2}
$$

for a suitable function $\eta: \mathbb{T} \rightarrow[0,1]$ and a sufficiently small $\varepsilon>0$.

One choice for the function $\eta$ is $\eta(\theta)=1-\left(\frac{M(\theta)}{\|M\|}\right)^{2}$. This function achieves its maximum value 1 when the transfer matrix vanishes and decays rapidly outside its zeroes.

Notice that

$$
\lambda_{\varepsilon}^{2}=\exp \left(\int_{\mathbb{T}} \log \left(M(\theta)^{2}+\varepsilon \eta(\theta)\right) d \theta\right) ;
$$

hence we consider $\varepsilon>0$ such that $\left|\lambda_{\varepsilon}\right|<1$ (notice that $\left|\lambda_{0}\right|<1$ ).

Copyright (c) by SIAM. Unauthorized reproduction of this article is prohibited. 
By defining

$$
\Lambda(\theta)=\frac{M(\theta)}{\sqrt{M(\theta)^{2}+\varepsilon \eta(\theta)}} \lambda_{\varepsilon}
$$

we obtain that $P_{1}, P_{2}=P_{1}^{-1}$, and $\Lambda$ satisfy equation

$$
P_{2}(\theta+\omega) M(\theta) P_{1}(\theta)=\Lambda(\theta) .
$$

Remark 4.2. In numerical computations these equations are solved by matching Fourier coefficients up to a finite order, even though the analytical solution of small divisors equations involve the smoothness of the transfer matrix and Diophantine properties of the rotation $\omega$. These are intermediate computations to produce initial data to be validated by our computer programs.

Numerical comparison of both methods. The Lyapunov metric method and the almost reducibility method have been tested, among others, for the period 2 attracting curve of the quasi-periodically driven logistic map with $D=0.1$ and $a=3.250$. In this case, the transfer matrix is noninvertible, hence nonreducible to constant. See Figure 6 to check differences between the two methods. In Figure 6(a) we can see that the dynamics of the linear cocycle is locally expanding in some regions (but it is globally contracting), while in Figures 6(e) and 6(c) the linear cocycles are locally and globally contracting. Notice that the Fourier coefficients of the reduced matrix $\Lambda(\theta)$, Figure 6(d), decay slowly when using the Lyapunov metric method, while they decay exponentially fast when using the almost reducibility method, Figure 6(f).

4.3. Computer validations. Motivated by the previous numerical study (see subsection section 4.1), we have validated the invariant curves appearing in the bifurcation diagram in Figure 3(a) up to values of $a$ close to the smooth bifurcations (A)-(B) (transcritical) and (D)-(E) (period doubling) and the nonsmooth bifurcation $(\mathrm{E})-(\mathrm{F})$. We report here in detail the existence of the repellor in regions $(\mathrm{E})$ and $(\mathrm{F})$ and the existence of the period 2 attracting curve near the nonsmooth bifurcation $(\mathrm{E})-(\mathrm{F})$.

Invariant curves in regions (A), (B), (C), and (D) have been validated using no more than 20 Fourier modes. The validations near the smooth bifurcations have been performed obtaining results similar to the ones reported below for the repellor.

To summarize the validations that we will present in detail, we have the proofs in the next subsections.

4.3.1. Proof of Proposition 1.1: Validation of the repellor. Here we explain the validation of the repelling curve. First, we validate analytically the existence of this curve for $a \in(4.6, \infty)$ and then, via computer-assisted proofs, we validate it for $a \in(3.157065,5)$ and check that the two families match.

Analytic validation. For the analytic validation, it is convenient to consider the following right inverse of $\left(F, t_{\omega}\right)$ :

$$
\begin{aligned}
\left(G, t_{-\omega}\right): & \mathbb{R} \times \mathbb{T} \\
(z, \theta) & \longrightarrow\left(\frac{1}{2}+\frac{1}{2} \sqrt{1-4 \frac{z}{a(1+D \cos (2 \pi(\theta-\omega)))}}, \theta-\omega\right) .
\end{aligned}
$$

We apply the validation algorithm with the following initial datum: $K(\theta)=\frac{a-1}{a}, P_{1}(\theta)=$ 


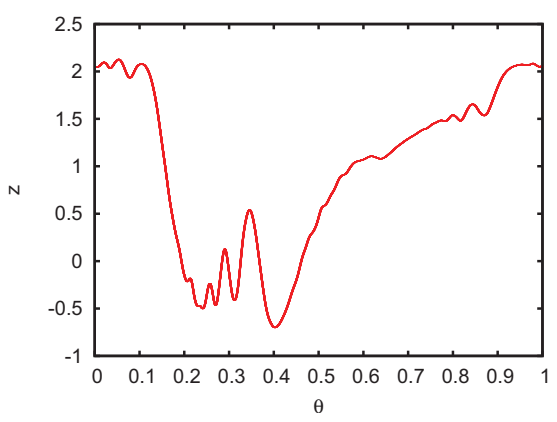

(a) Transfer matrix $M(\theta)$.

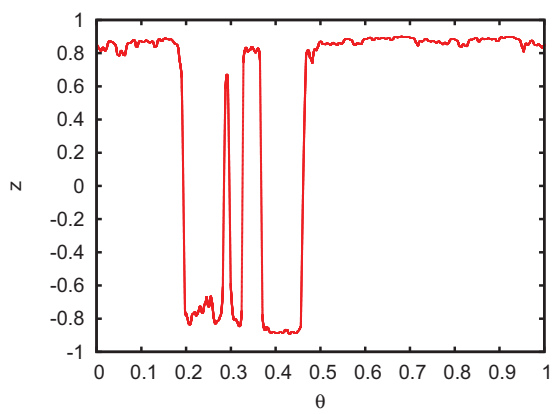

(c) Reduced matrix $\Lambda(\theta)$, computed via Lyapunov metric method.

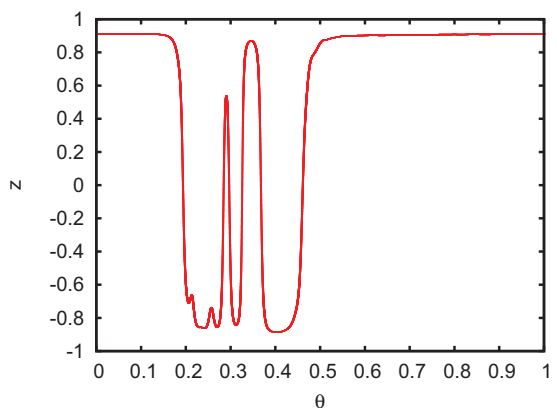

(e) Reduced matrix $\Lambda(\theta)$, computed via almost-reducibility method.

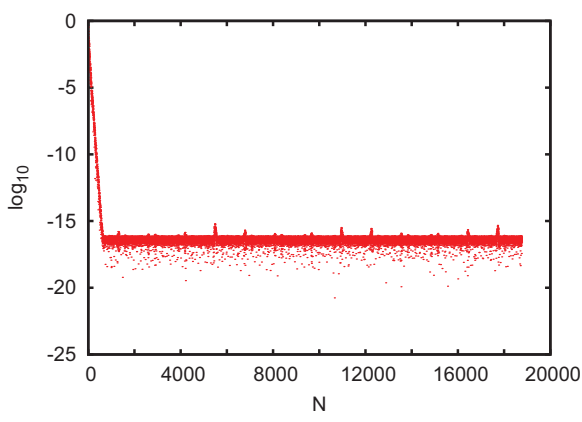

(b) Modes of the transfer matrix $M(\theta)$.

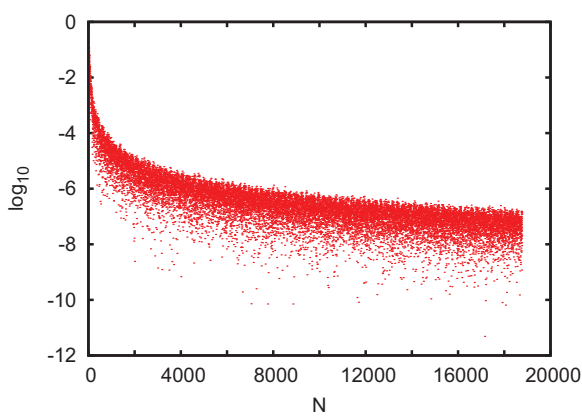

(d) Modes of the reduced matrix $\Lambda(\theta)$, computed via Lyapunov metric method.

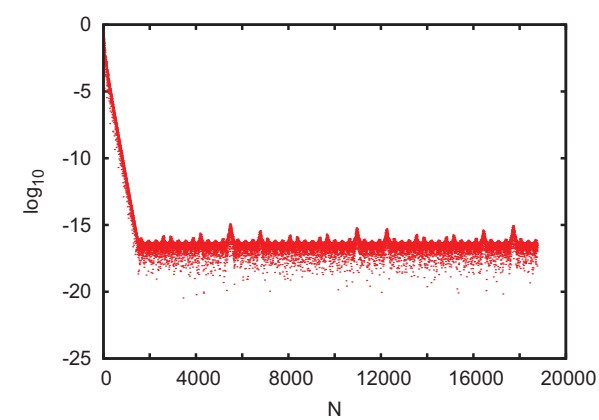

(f) Modes of the reduced matrix $\Lambda(\theta)$, computed via almost-reducibility method.

Figure 6. Graphical comparison of the computed reduced $\Lambda(\theta)$ of the period 2 attracting curve for a $=3.25$ and $D=0.1$.

$P_{2}(\theta)=1, \Lambda(\theta)=M(\theta)=\mathrm{D}_{z} G\left(\frac{a-1}{a}, \theta\right)$. In the following, we consider the bound

$$
\Delta=\sqrt{1-\frac{4(a-1)}{a^{2}(1-D)}}
$$

The constants $\rho=\frac{1}{2}-\frac{1}{a}-\frac{1}{2} \Delta, \sigma=\tau=0$, and $\lambda=\hat{\lambda}=\frac{1}{a(1-D) \Delta}$ satisfy inequalities $(2.1)$, (2.2), (2.3), and (2.4) of the validation Theorem 2.8. Inequality (2.5) is satisfied if $a>\frac{1}{(1-D) \Delta}$. 
Choosing $r=\frac{2 \rho}{1-\lambda}$, we obtain the upper bound of the second derivative (3.1) to be

$$
b=\frac{2}{a^{2}(1-D)^{2}\left(1-\frac{4\left(\frac{a-1}{a}+r\right)}{a(1-D)}\right)^{\frac{3}{2}}},
$$

from which we obtain $h=(1-\lambda)^{-2} b \rho$. Fixing $D$ for $a>0$ sufficiently big, we obtain $h<\frac{1}{2}$ and then there is a unique invariant torus close to initial data $K$. In particular, for $D=0.1$, we obtain the crude lower bound $a>4.6$ (for which $h<0.45$ ).

Computer validation. After showing the existence of the repelling curve for values $a>4.6$, we proved (computer-assisted) the existence of the family of the repelling curve for $3.157065 \leq$ $a \leq 5$. At $a=5$ the Lyapunov multiplier is 2.962531 , while at the end of the validation, $a=3.157065$, the Lyapunov multiplier is 1.016861. This validation has been done, using expression (4.1), by computing the initial data using the algorithms presented in subsection section 4.2 with 30 Fourier modes (This choice of number of modes is done in order to ensure that the discarded modes are of magnitude less than $10^{-8}$.) We emphasize that the width of the intervals of validation shorten as they approach to the period doubling bifurcation value $a \simeq 3.143$. The algorithm stops when the width of the intervals is less that $10^{-6}$, reaching $a=3.157065$. See Figure 7(a).

Remark 4.3. In this computation we apply the validation algorithm 2800 times and the time of computation is around 307 minutes. This means that each validation step, which consists in computing the initial data, validating the existence and uniqueness of a FHIT near it, and then checking the matching, takes around 6.5 seconds.

In order to show how the upper bounds of the validation algorithm behave near the bifurcation value, we apply the validation algorithm for values $a=3.16+0.01 \cdot j$ with $j=0, \ldots, 184$, using 30 Fourier modes. The results are displayed in Figures 7(b), 7(c), and 7(d).

Remark 4.4. While the numerically computed initial data is produced with a nonrigorous estimate of the error of order $10^{-14}$, and although the validations are done using the FILIB ++ library, which operates with intervals in double precision, the rigorous error bounds achieve order $10^{-10}$.

4.3.2. Proof of Proposition 1.1: Validation of the nonreducible period 2 attracting curve. The goal in this subsection is to validate nonreducible period 2 attracting curves near the predicted nonsmooth bifurcation value $a_{*} \approx 3.271$. To do so, we considered the second iterate of the driven logistic map (4.1):

$$
\begin{aligned}
\left(F, t_{\omega}\right)^{2}: & \mathbb{R} \times \mathbb{T} \longrightarrow \mathbb{R} \times \mathbb{T}, \\
(z, \theta) \longrightarrow & (F(F(z, \theta), \theta+\omega), \theta+2 \omega) .
\end{aligned}
$$

First, we perform a numerical study of the regularity of the initial data: the torus $K$, the transformations $P_{1}$ and $P_{2}$, and the normalized cocycle $\Lambda$. Since the associated transfer matrix $M$ is noninvertible, we use the almost-reducibility method to compute $P_{1}, P_{2}$, and $\Lambda$. Figure 8 shows, with respect to parameter $a$, a numerical estimate of the maximum slope of the computed initial data. Note that $P_{1}$ is the initial datum with the biggest slope. For example, at $a=3.265$ the slope of $P_{1}$ is $4.3 \cdot 10^{4}$, while the slopes of the torus and the 


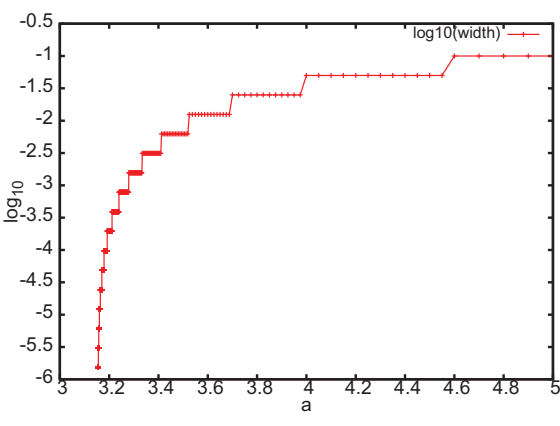

(a) Width of the intervals of the family validation.

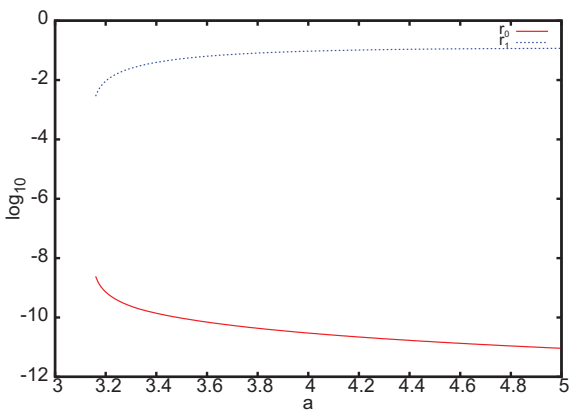

(c) $r_{0}$ and $r_{1}$ values of the validations.

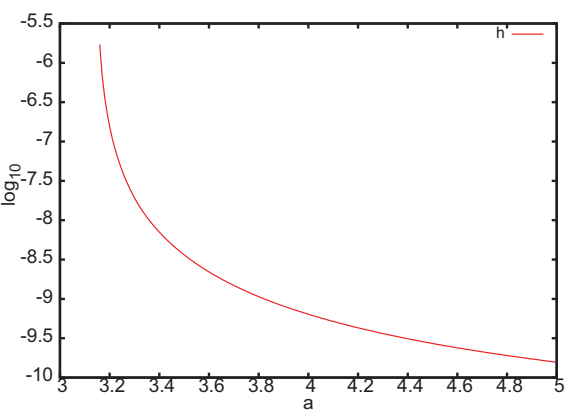

(b) $h$ value of the validations.

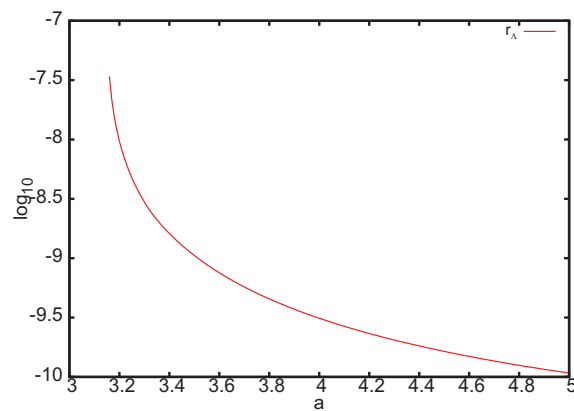

(d) $r_{\Lambda}$ error values.

Figure 7. Data obtained of the validations of the repelling curve for $D=0.1$.

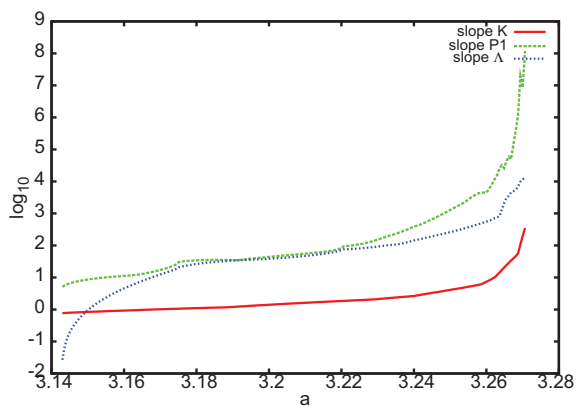

Figure 8. Maximum slopes of the period 2 attracting curve $K$ (in red), its $P_{1}$ transformation (in green), and the normalized cocycle $\Lambda$ (in blue) with respect to parameter a.

normalized cocycle are $2.4 \cdot 10^{1}$ and $3.07 \cdot 10^{3}$, respectively. Notably, at $a=3.269$ the slope of $P_{1}$ is $4.25 \cdot 10^{6}$. Hence, $P_{1}$ is used in order to determine the number of Fourier modes in the validation process, because it is the initial datum with the biggest Fourier coefficients. We choose the number of modes so that the discarded ones are of magnitude less than $10^{-8}$. Figure 9 shows the initial data $K$ (and $M$ ), $P_{1}$ and $\Lambda$ for $a=3.265$ and $a=3.269$. Notice that a small change in the value of $a$ leads to a dramatic change in the initial data.

The validation results for different values of the parameter $a$ are shown in Table 1 . The initial data used as input is computed with high accuracy because at these parameter values,

Copyright $@$ by SIAM. Unauthorized reproduction of this article is prohibited. 


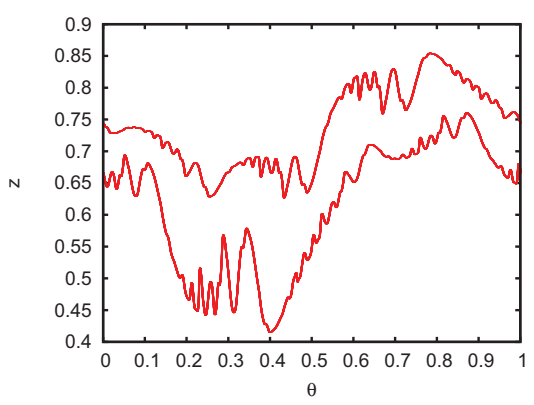

(a) Torus $K(\theta)$ and its image for $a=$ 3.265 .

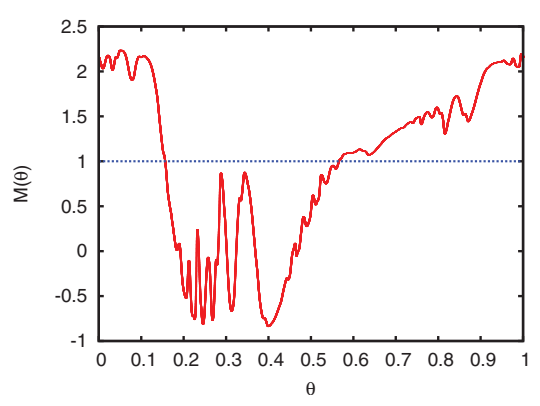

(c) Cocycle $M(\theta)$ for $a=3.265$.

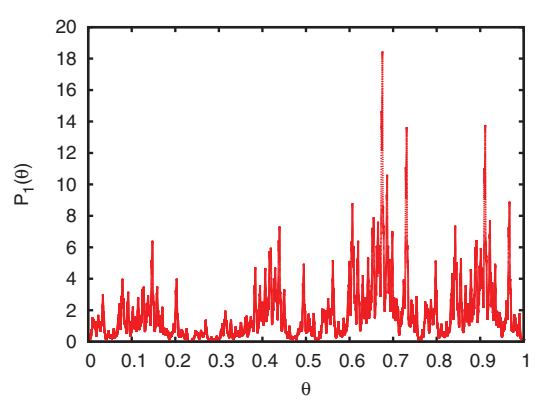

(e) Transformation $P_{1}(\theta)$ for $a=$ 3.265 .

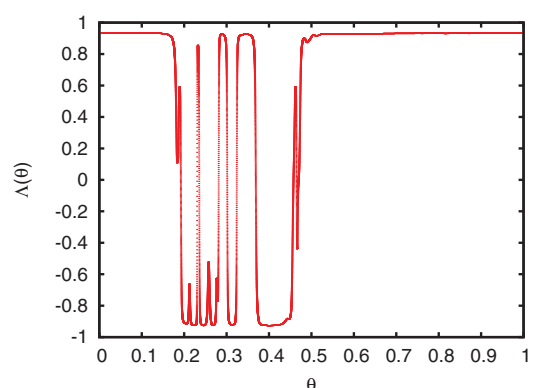

(g) Reduced cocycle $\Lambda(\theta)$ for $a=$ 3.265 .

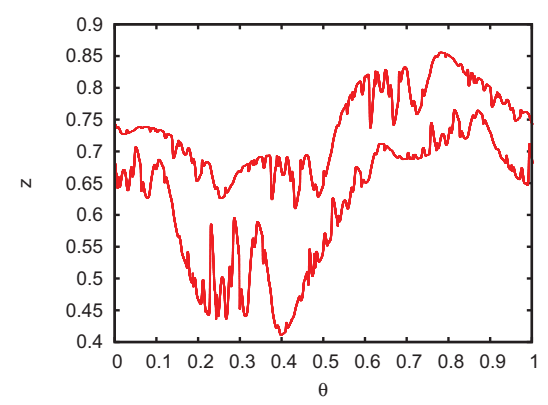

(b) Torus $K(\theta)$ and its image for $a=$ 3.269 .

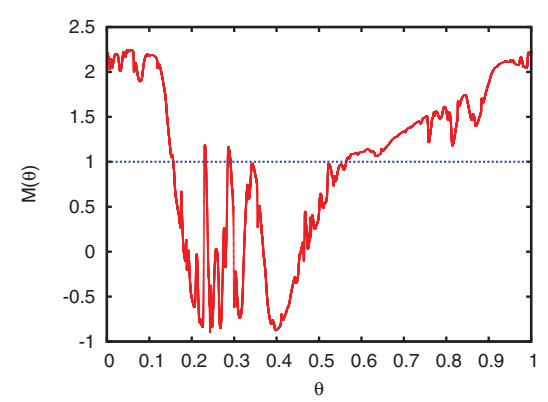

(d) Cocycle $M(\theta)$ for $a=3.269$.

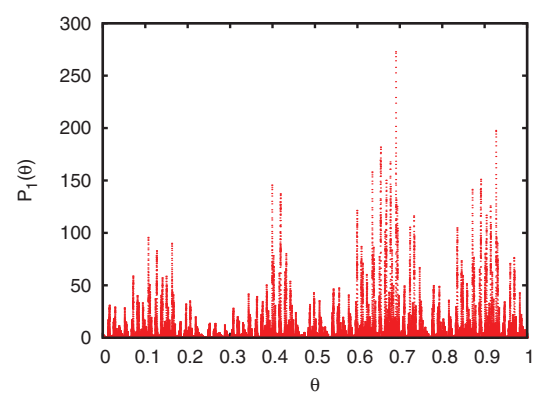

(f) Transformation $P_{1}(\theta)$ for $a=$ 3.269 .

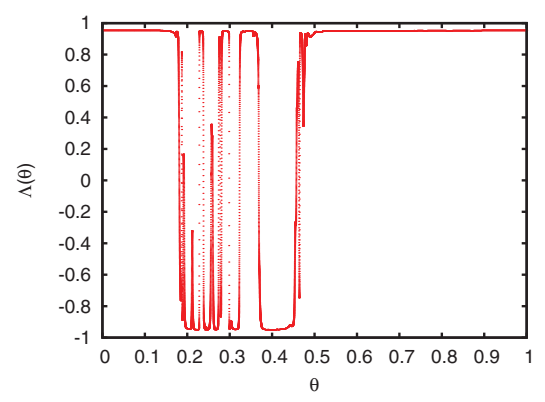

(h) Reduced cocycle $\Lambda(\theta)$ for $a=$ 3.269 .

Figure 9. Graphs of the initial data close to the breakdown of the period 2 curve. 
Table 1

Validation results of the period 2 invariant torus of the driven logistic map for different values of a close to breakdown.

\begin{tabular}{|c||c|c|c|}
\hline \hline$a$ & 3.265 & 3.268 & 3.269 \\
\hline$h$ & $3.046383 \mathrm{e}-05$ & $2.248226 \mathrm{e}-03$ & $4.203495 \mathrm{e}-01$ \\
\hline$r_{0}$ & $5.365990 \mathrm{e}-09$ & $1.701127 \mathrm{e}-07$ & $3.635973 \mathrm{e}-06$ \\
\hline$r_{\Lambda}$ & $5.815762 \mathrm{e}-03$ & $4.542701 \mathrm{e}-04$ & - \\
\hline Order & 3000 & 17,000 & 27,000 \\
\hline Time (minutes) & 5 & 130 & 361 \\
\hline
\end{tabular}

the period 2 attracting curve is near the repellor curve. Note also that in all these validations the time computation depends heavily on the regularity of the initial data, because less regularity implies the use of more Fourier modes to represent the initial data, which implies more computational time.

Remark 4.5. Note that in Table 1, for the parameter value $a=3.269$, we do not have an estimation of $r_{\Lambda}$. This is because the validation algorithm cannot compute it due to the fact that the upperbound $\mu$ (see point (5.2) of Theorem 2.8) is bigger than $\frac{1}{4}$. This means that although we could validate the existence (and local uniqueness) of the period 2 invariant torus, we could not validate the distance between the initial data $\Lambda_{0}$ and the transfer operator of the truly invariant torus of the system.

5. Example 2: Computer validations on the verge of the hyperbolicity breakdown of a saddle torus. In this section we report computer validations of existence of saddle tori on the verge of their hyperbolicity breakdown for the quasi-periodically forced standard map (1.2). This phenomenon was described in $[29,26]$ for similar models.

5.1. Numerical exploration. For every $\kappa>0$, there exists a family $K_{\varepsilon}(\theta)$ of saddle type invariant tori with $\varepsilon \in\left[0, \varepsilon_{c}\right)$, and $K_{0}(\theta)=\left(\frac{1}{2}, 0\right)$. These invariant tori and their invariant bundles are computed using Fourier methods [27].

An interesting problem is to approach as closely as possible the limiting value $\varepsilon_{c}$, the critical parameter value, and study the obstructions to fiberwise hyperbolicity. We perform a numerical exploration and we find that the bifurcation mechanism around $\varepsilon_{c}$ depends on $\kappa$. Particular examples are as follows:

(i) For $\kappa=0.3$, then $\varepsilon_{c} \approx 1.3364054$, and there is a smooth bifurcation: the hyperbolicity is lost because the maximal Lyapunov multiplier goes to 1 as $\varepsilon$ goes to $\varepsilon_{c}$, but the invariant subbundles collide smoothly. Also, the invariant tori are smooth at the bifurcation. Similar behavior happens for close values of $\kappa$.

(ii) For $\kappa=1.3$, then $\varepsilon_{c} \approx 1.2352755$, and there is a nonsmooth bifurcation: the hyperbolicity is broken down because the invariant bundles collide nonuniformly as $\varepsilon$ goes to $\varepsilon_{c}$, and the maximal Lyapunov multiplier stays far from 1 . The invariant tori loose their smoothness at the bifurcation. Similar behavior happens for close values of $\kappa$.

In this paper we report results for $\kappa=1.3$. Figure 10 shows the observables (Lyapunov multiplier and minimum distance between the invariant bundles) near the breakdown. Figure 2 shows the invariant tori and their invariant bundles for several values of the parameter $\varepsilon$.

Copyright $\odot$ by SIAM. Unauthorized reproduction of this article is prohibited. 


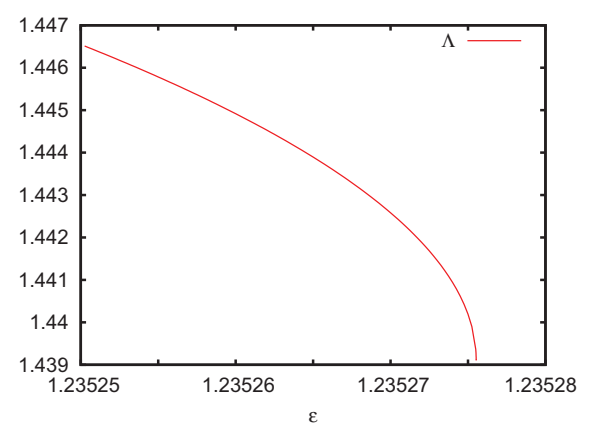

(a) Lyapunov multiplier as a function of $\varepsilon$.

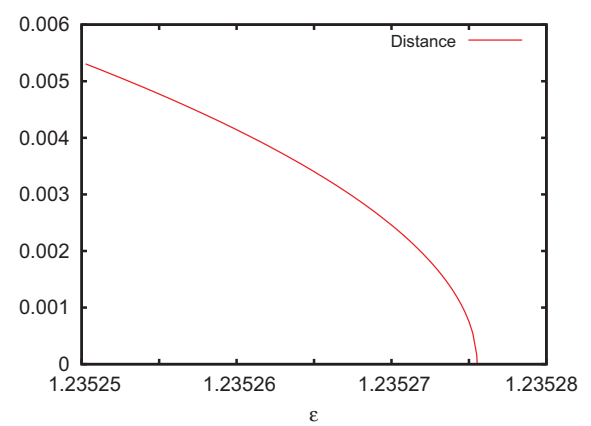

(b) Minimum distance between subbundles. It approaches 0 as $\varepsilon$ gets close to $\varepsilon_{c}$.

Figure 10. Nonsmooth bifurcation: observables for $\kappa=1.3$ near the bifurcation value $\varepsilon_{c} \approx 1.2352755$.

Table 2

Numerical estimates (nonrigorous) of the bounds of the validation algorithm for the initial data producing the validated results.

\begin{tabular}{|c||c|c|c|}
\hline$\varepsilon$ & 1.235270 & 1.235273 & 1.235275 \\
\hline \hline$h$ & $2.169418 \mathrm{e}-08$ & $1.260415 \mathrm{e}-05$ & $8.546872 \mathrm{e}-06$ \\
\hline$r_{0}$ & $1.801702 \mathrm{e}-14$ & $5.751160 \mathrm{e}-12$ & $1.234738 \mathrm{e}-12$ \\
\hline$r_{1}$ & $1.661000 \mathrm{e}-06$ & $9.125708 \mathrm{e}-07$ & $2.889308 \mathrm{e}-07$ \\
\hline$\mu$ & $7.453060 \mathrm{e}-08$ & $4.723001 \mathrm{e}-05$ & $1.346137 \mathrm{e}-05$ \\
\hline$r_{\Lambda}$ & $2.901342 \mathrm{e}-08$ & $1.831964 \mathrm{e}-05$ & $5.199567 \mathrm{e}-06$ \\
\hline Order & 8192 & 8192 & 16,384 \\
\hline
\end{tabular}

As an illustration of the numerical computations on the verge of the breakdown, Table 2 shows the estimated values of the bounds of the validation algorithm for several values of the parameter $\varepsilon$ close to $\varepsilon_{c}$.

5.2. Computer validations. In this section we report computer validations of the invariant tori for the nonsmooth bifurcation scenario for $\kappa=1.3$ with $\varepsilon_{c}=1.2352755$. This is a challenging example because the invariant subbundles near the bifurcation are quite wild (SNA behavior in the projectivized cocycle; see [30]). Thousands of Fourier modes are needed in order to obtain good initial data for the validation algorithm.

5.2.1. Proof of Proposition 1.2. For proving point (i) in Proposition 1.2, we validate tori $K_{\varepsilon}$ for all the values of $\varepsilon$ in the proposition. Note that the difference between the predicted breakdown value $\varepsilon_{c}$ and the last validation $\varepsilon=1.2352$ is less than of order $8 \cdot 10^{-5}$. These results are reported in Figure 11. We observe that as $\varepsilon$ increases, the upper bounds of the validation algorithm $h$ and $r_{0}$, which measure the quality of the approximate invariant torus, increase, while the lower bound of $r_{1}$, which measures the size of the uniqueness strip, decreases. We also observe that the upper bounds $\mu$ and $r_{\Lambda}$, which measure the quality of the approximate invariant bundles, increase. The number of Fourier modes required in the validations goes from 0 to 1280.

We use the validation algorithm for families of FHIT to prove point (ii) in Proposition 1.2. The validations in the parameter interval $\varepsilon \in[0,1.167434]$ have been performed with Fourier 


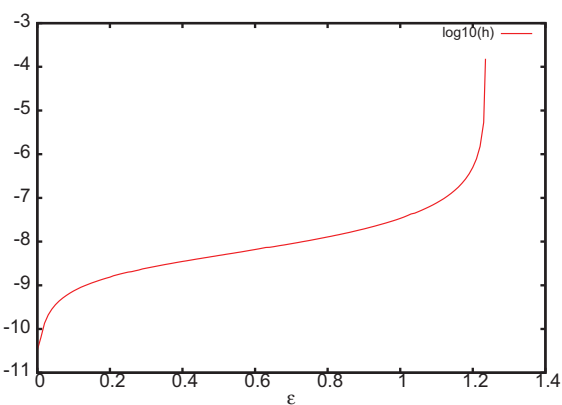

(a) $h$ value of Newton-Kantorovich theorem.

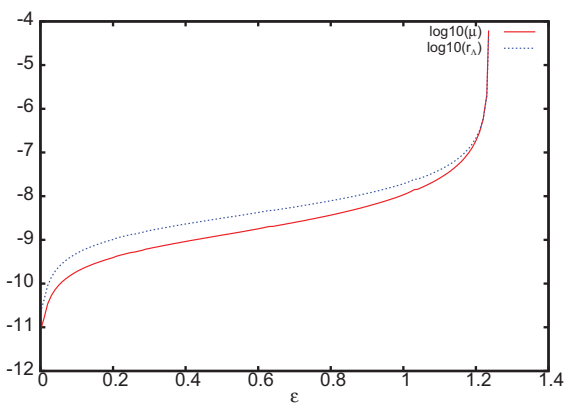

(c) Quality of the invariant bundles: $\mu$ and $r_{\Lambda}$

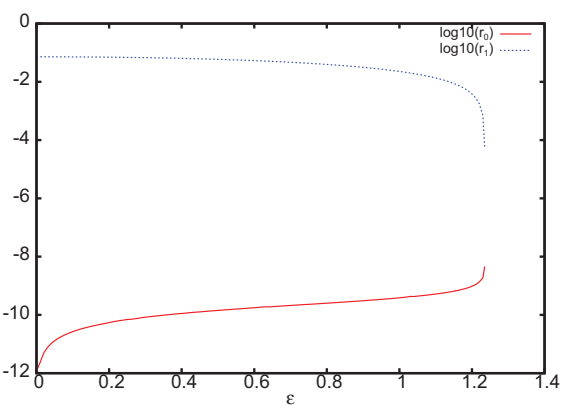

(b) Quality of invariant tori: $r_{0}$ and $r_{1}$.

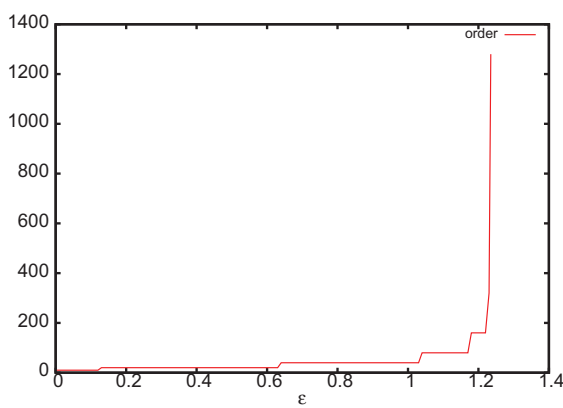

(d) Order of Fourier model.

Figure 11. Data output obtained from the validations of the invariant tori and their invariant bundles for $\kappa=1.3$ with respect to $\varepsilon$. See text for details.

models of order less than 2000. The main problem for validating the family further is that the width of the parameter intervals required in the algorithm is too small, of order $10^{-6}$. This happens because as the family approaches the bifurcation, the invariant tori and their associated initial data $\left(P_{1}, P_{2}\right.$ and $\Lambda$ ) changes dramatically. (See, for example, the behavior of the Lyapunov multiplier in Figure 10.)

Finally, we prove point (iii) in Proposition 1.2. The validation of the initial data for the values $\varepsilon=1.235270,1.235273,1.235275$, with Lyapunov multipliers $\Lambda=1.442582,1.441463$, 1.440193 , illustrates the applicability of the validation algorithm in cases that are extremely close to the nonsmooth bifurcation. The obtained results are shown in Table 3. Note that the difference between 1.235275 and the predicted bifurcation value, 1.2352755 , is less than $5.3 \cdot 10^{-7}$.

6. Final comments. In the validation examples we show that it is important to understand the dynamics around the invariant tori in order to obtain successful validations. A key role is played by the matrix-valued maps $P_{1}$ and $P_{2}$, which give an adapted frame where the hyperbolicity conditions are checked, and the hyperbolicity constant $\lambda$. In fact, the condition number $\frac{\left\|P_{1}\right\|\left\|P_{2}\right\|}{1-\lambda}$ gives a measure of the quality of the hyperbolicity. The bigger it is, the harder the validations. Note that this condition number is big when the invariant torus is

Copyright (C) by SIAM. Unauthorized reproduction of this article is prohibited. 
Table 3

Validation results of invariant tori of the quasi-periodically forced standard map for three $\varepsilon$ values near the predicted breakdown. Note that the order of the Fourier models and the time of validation increase as $\varepsilon$ increases. Compare these rigorous results with the nonrigorous estimates given in Table 2.

\begin{tabular}{|c||c|c|c|}
\hline$\varepsilon$ & 1.235270 & 1.235273 & 1.235275 \\
\hline \hline$h$ & $2.853269 \mathrm{e}-03$ & $8.140590 \mathrm{e}-03$ & $8.928078 \mathrm{e}-02$ \\
\hline$r_{0}$ & $1.302039 \mathrm{e}-07$ & $2.490723 \mathrm{e}-07$ & $1.035418 \mathrm{e}-06$ \\
\hline$r_{1}$ & $9.100589 \mathrm{e}-05$ & $6.069352 \mathrm{e}-05$ & $2.107294 \mathrm{e}-05$ \\
\hline$\mu$ & $1.825306 \mathrm{e}-03$ & $5.188943 \mathrm{e}-03$ & $3.841927 \mathrm{e}-02$ \\
\hline$r_{\Lambda}$ & $1.370355 \mathrm{e}-03$ & $3.900239 \mathrm{e}-03$ & $2.985134 \mathrm{e}-02$ \\
\hline Order & 5802 & 7918 & 27,692 \\
\hline Time (minutes) & 103 & 154 & 1094 \\
\hline
\end{tabular}

near the boundaries of the uniform hyperbolicity. Moreover, the computation of the adapted frame is difficult in cases in which the dynamics is nonreducible or when the torus is about to break.

In the quasi-periodically driven logistic map, we saw that in order to validate the existence of the period 2 attracting curve it is important to establish whether the linearized dynamics around the torus is reducible. The linear behavior determines the possible adapted frames $P_{1}$, $P_{2}$ and also the possible parameterization of the dynamics on the bundles, given by $\Lambda$. The nonreducible case is the most difficult case to work on. We used different methods to study this case and then compared them. Finally, we applied it to see the results and effectiveness of the validation, obtaining good results for accuracy. In the quasi-periodically forced standard map, we saw that the linearized dynamics, as long as the FHIT exists, is reducible, but when the invariant tori approach a nonsmooth breakdown, we observed that this reducibility condition blows up. We therefore studied the effectiveness of the validation algorithm near this blow-up and obtained that the validation algorithm can be applied near the breakdown and thus deals successfully with this singular behavior.

We emphasize that our methodology can be applied to many other examples for validating invariant tori and produce rigorous bounds on the thresholds, from smooth bifurcations (saddle-node, period doubling, Hamiltonian saddle-node) to nonsmooth bifurcations or breakdowns (nonsmooth saddle-node and nonsmooth pitchfork bifurcations to SNA [24, 35, 54], SNAs in Harper maps), and also for computing enclosures of the spectrum Schrödinger operators $[30,29]$. The examples reported in this paper present certain characteristics that make them more difficult to deal with. The bifurcating objects of the SNA mechanisms mentioned above are attracting and reducible tori, while in the Heagy-Hammel mechanisms reported in this paper the attracting tori are nonreducible. We emphasize that nonreducibility is an essential feature of some fractalization mechanisms producing false SNA [10, 31, 37], but computer-assisted proofs in those cases are even more challenging since the tori wrinkle substantially.

The nonsmooth Hamiltonian saddle-node mechanism of a saddle torus is difficult to deal with. For instance, the smooth Hamiltonian bifurcation in which a saddle torus smoothly bifurcates to an elliptic torus has been observed in the quasi-periodically forced standard map for $\kappa=0.3$. In this case the validation can be easily carried out up to values close to

Copyright $\odot$ by SIAM. Unauthorized reproduction of this article is prohibited. 
the estimated critical value $\varepsilon_{c}$ using less than a hundred Fourier modes, in contrast to the thousands of Fourier modes needed to validate invariant tori close to nonsmooth breakdowns.

The computational time of the validation algorithms depends heavily on the regularity of the initial data and hence their number of Fourier modes. The most time-consuming computations with Fourier models are the product and the evaluation. Although the times reported in this paper correspond to computations with a single processor, we have also used the library OpenMP (see [14]) in order to have parallel computations (by distributing the product and evaluation routines on the processors).

All the validations were performed using double precision with the aid of the interval package library FILIB++.This, of course, has its limitations, and if we want to validate invariant tori more precisely, then we will need a multiprecision library, but the procedure for validating the invariant tori remains the same. An example where a multiprecision library is needed is [12].

The models worked out in this paper have simple analytic expressions. But our validation algorithms can be applied to more general models, as long as we can evaluate the map (and its first and second derivatives). For instance, for a skew product flow, we can consider its Poincaré map with the variationals [5, 63].

Appendix A. Operations with Fourier models. Here we detail some implementations of compositions with Fourier models with elementary functions that are combinations of finitely many arithmetic operations and compositions with simple functions (or intrinsic functions [56]) such as the power function, the exponential function, or the trigonometric functions. Since we have to truncate the results, let us start with the following definition.

Definition A.1. Let $\hat{G}=(G(\theta), R)$ be a Fourier model of order $m$, and $\ell \geq 0$. We define the $\ell$-tail of $\hat{G}$ as $\hat{G}_{>\ell}=\left(G_{>\ell}(\theta), R\right)$, where $G_{>\ell}(\theta)$ is the intervalar Fourier polynomial $\sum_{k=\ell+1}^{m}\left(A_{k} \cos (2 \pi k \theta)+B_{k} \sin (2 \pi k \theta)\right)$. We define the $\ell$-enclosure of $\hat{G}$ as $\hat{G}_{\leq \ell}=$ $\left(G_{\leq \ell}, \hat{G}_{>\ell}([0,1])\right)$, where $G_{\leq \ell}(\theta)=A_{0}+\sum_{k=1}^{\ell}\left(A_{k} \cos (2 \pi k \theta)+B_{k} \sin (2 \pi k \theta)\right)$. Abusing notation, $\hat{G}_{>-1}=\hat{G}, \hat{G}_{\leq-1}=\hat{G}([0,1])$.

The arithmetic operations with Fourier models are defined as follows. Addition and subtraction of two Fourier models $\hat{G}$ and $\hat{H}$ is defined componentwise:

$$
\hat{G}+\hat{H}=(G(\theta)+H(\theta), R(\hat{G})+R(\hat{H})), \hat{G}-\hat{H}=(G(\theta)-H(\theta), R(\hat{G})-R(\hat{H})) .
$$

If $J$ is an interval, we define the multiplication of $\hat{G}$ with $J$ as

$$
J \cdot \hat{G}=(J G(\theta), J R) .
$$

The product of $\hat{G}$ and $\hat{H}$ is

$$
\hat{G} \cdot \hat{H}=(G(\theta) H(\theta), G([0,1]) R(\hat{H})+H([0,1]) R(\hat{G})+R(\hat{G}) R(\hat{H})) .
$$

In order to bound the order of the Fourier models through the operations in a computation, we in fact compute enclosures of the products. For instance, if $\hat{G}$ and $\hat{H}$ are two Fourier models of order $m$, their $m$-product is the $m$-enclosure of the product, i.e., $(\hat{G} \cdot \hat{H})_{\leq m}$.

Once we have defined the arithmetic operations with Fourier models, compositions with polynomials are straightforward. Enclosures of the compositions of Fourier models with simple

Copyright (c) by SIAM. Unauthorized reproduction of this article is prohibited. 
functions, such as the exponential, power function, logarithm, etc. can be performed with the aid of the corresponding Taylor polynomial approximations (and Lagrange error bounds). We explain here the composition with the sine and cosine functions, which are the ones that appear in our examples.

Given $\ell>0$, let $S_{\ell}(x), C_{\ell}(x)$ be the Taylor polynomials of degree $\ell$ of the sine and cosine functions, respectively. Let

$$
\hat{S}_{\ell}(x)=S_{\ell}(x)+\frac{[-1,1]}{(\ell+1) !} x^{l+1}, \hat{C}_{\ell}(x)=C_{\ell}(x)+\frac{[-1,1]}{(\ell+1) !} x^{l+1}
$$

be the corresponding polynomials with Lagrange error bounds. Then, the compositions of $\left(F, t_{\omega}\right)$ with the sine and cosine functions are enclosed in

$$
\begin{aligned}
& \sin _{\ell}(\hat{G})=\sin \left(A_{0}\right) \cdot \hat{C}_{\ell}\left(\hat{G}_{>0}\right)+\cos \left(A_{0}\right) \cdot \hat{S}_{\ell}\left(\hat{G}_{>0}\right), \\
& \cos _{\ell}(\hat{G})=\cos \left(A_{0}\right) \cdot \hat{C}_{\ell}\left(\hat{G}_{>0}\right)-\sin \left(A_{0}\right) \cdot \hat{S}_{\ell}\left(\hat{G}_{>0}\right),
\end{aligned}
$$

respectively. In computer implementations, the order $\ell$ of the Taylor polynomials is chosen such that $\left|\frac{1}{(l+1) !}\left(\hat{G}_{>0}[0,1]\right)^{l+1}\right|$ is less than a given tolerance. We also use $m$-products in the intermediate computations.

Another operation used in this paper is the shift of a Fourier model $\hat{G}=(G(\theta), R)$ by an (interval) rotation $\omega$. This is the Fourier model $S_{\omega}(\hat{G})=(S(\theta), R)$ with $S(\theta)=A_{0}+$ $\sum_{k=1}^{m}\left(A_{k}^{\prime} \cos (2 \pi k \theta)+B_{k}^{\prime} \sin (2 \pi k \theta)\right)$, where

$$
A_{k}^{\prime}=B_{k} \cos (2 \pi k \omega)-A_{k} \sin (2 \pi k \omega), B_{k}^{\prime}=A_{k} \cos (2 \pi k \omega)+B_{k} \sin (2 \pi k \omega) .
$$

Acknowledgments. We thank Maciej Capiński, Àngel Jorba, Rafael de la Llave, and Carles Simó for their comments and fruitful discussions on various aspects of this research. We thank Alejandra González and Pere Gomis for proofreading a previous version of this paper. We thank the developers of the rigorous interval library FILIB++.

\section{REFERENCES}

[1] R. A. Adomaitis And I. G. Kevrekidis, Noninvertibility and the structure of basins of attraction in a model adaptive control system, J. Nonlinear Sci., 1 (1991), pp. 95-105.

[2] R. A. Adomaitis, I. G. Kevrekidis, And R. DE la Llave, A computer-assisted study of global dynamic transitions for a noninvertible system, Internat. J. Bifur. Chaos Appl. Sci. Engrg., 17 (2007), pp. 13051321.

[3] D. V. Anosov, Geodesic flows on closed Riemann manifolds with negative curvature, Proc. Steklov Inst. Math., 90 (1967).

[4] G. Arioli And H. Koch, Integration of dissipative partial differential equations: A case study, SIAM J. Appl. Dyn. Syst., 9 (2010), pp. 1119-1133.

[5] M. BERZ AND K. MAKInO, Verified integration of ODEs and flows using differential algebraic methods on high-order Taylor models, Reliab. Comput., 4 (1998), pp. 361-369.

[6] Z. I. Bezhaeva And V. I. Oseledets, On an example of a "strange nonchaotic attractor," Funktsional. Anal. i Prilozhen., 30 (1996), pp. 1-9, 95.

[7] K. BJERKLÖv, SNA's in the quasi-periodic quadratic family, Comm. Math. Phys., 286 (2009), pp. $137-161$.

[8] H. W. Broer, A. Hagen, And G. Vegter, Numerical continuation of normally hyperbolic invariant manifolds, Nonlinearity, 20 (2007), pp. 1499-1534.

Copyright $\odot$ by SIAM. Unauthorized reproduction of this article is prohibited. 
[9] H. W. Broer, H. M. Osinga, And G. Vegter, Algorithms for computing normally hyperbolic invariant manifolds, Z. Angew. Math. Phys., 48 (1997), pp. 480-524.

[10] H. W. Broer, C. Simó, And R. Vitolo, Chaos and quasi-periodicity in diffeomorphisms of the solid torus, Discrete Contin. Dyn. Syst. Ser. B, 14 (2010), pp. 871-905.

[11] M. J. CAPIŃSKI, Covering relations and the existence of topologically normally hyperbolic invariant sets, Discrete Contin. Dyn. Syst., 23 (2009), pp. 705-725.

[12] M. J. Capiński And C. Simó, Computer Assisted Proof for Normally Hyperbolic Invariant Manifolds, Nonlinearity, to appear.

[13] A. Celletti And L. Chierchia, KAM stability and celestial mechanics, Mem. Amer. Math. Soc., 187 (2007).

[14] B. Chapman, G. Jost, and R. van Der Pas, Using OpenMP: Portable Shared Memory Parallel Programming (Scientific and Engineering Computation), MIT Press, Cambridge, MA, 2007.

[15] C. Chicone and Y. Latushkin, Evolution Semigroups in Dynamical Systems and Differential Equations, Math. Surveys Monogr. 70, American Mathematical Society, Providence, RI, 1999.

[16] R. DE LA LLAVE, Hyperbolic dynamical systems and generation of magnetic fields by perfectly conducting fluids, Geophys. Astrophys. Fluid Dyn., 73 (1993), pp. 123-131.

[17] R. DE la Llave, A tutorial on KAM theory, in Smooth Ergodic Theory and Its Applications (Seattle, WA, 1999), Proc. Sympos. Pure Math. 69, American Mathematical Society, Providence, RI, 2001.

[18] R. DE la Llave And D. Rana, Accurate strategies for small divisor problems, Bull. Amer. Math. Soc. (N.S.), 22 (1990), pp. 85-90.

[19] R. DE LA Llave AND D. RanA, Accurate strategies for K.A.M. bounds and their implementation, in Computer Aided Proofs in Analysis (Cincinnati, OH, 1989), IMA Vol. Math. Appl. 28, Springer, New York, 1991.

[20] J.-P. Eckmann, H. Koch, And P. Wittwer, A computer-assisted proof of universality for areapreserving maps, Mem. Amer. Math. Soc., 47 (1984).

[21] N. Fenichel, Persistence and smoothness of invariant manifolds for flows, Indiana Univ. Math. J., 21 (1971/1972), pp. 193-226.

[22] U. Feudel, S. Kuznetsov, and A. Pikovsky, Strange Nonchaotic Attractors, World Sci. Ser. Nonlinear Sci. Ser. A Monogr. Treatises 56, World Scientific Publishing, Hackensack, NJ, 2006.

[23] M. GameIro AND J.-P. LeSSARD, Analytic estimates and rigorous continuation for equilibria of higherdimensional PDEs, J. Differential Equations, 249 (2010), pp. 2237-2268.

[24] P. Glendinning, U. Feudel, A. S. Pikovsky, and J. Stark, The structure of mode-locked regions in quasi-periodically forced circle maps, Phys. D, 140 (2000), pp. 227-243.

[25] C. Grebogi, E. Ott, S. Pelikan, And J. A. Yorke, Strange attractors that are not chaotic, Phys. D, 13 (1984), pp. 261-268.

[26] A. Haro and R. DE la Llave, Manifolds on the verge of a hyperbolicity breakdown, Chaos, 16 (2006), 013120 .

[27] A. Haro AND R. DE la Llave, A parameterization method for the computation of invariant tori and their whiskers in quasi-periodic maps: Numerical algorithms, Discrete Contin. Dyn. Syst. Ser. B, 6 (2006), pp. 1261-1300.

[28] A. Haro AND R. DE la Llave, A parameterization method for the computation of invariant tori and their whiskers in quasi-periodic maps: Rigorous results, J. Differential Equations, 228 (2006), pp. 530-579.

[29] A. Haro AND R. DE la Llave, A parameterization method for the computation of invariant tori and their whiskers in quasi-periodic maps: Explorations and mechanisms for the breakdown of hyperbolicity, SIAM J. Appl. Dyn. Syst., 6 (2007), pp. 142-207.

[30] A. Haro And J. PUig, Strange nonchaotic attractors in Harper maps, Chaos, 16 (2006), 033127.

[31] A. Haro And Simó, To Be or Not To Be a SNA: That Is the Question, http://www.maia.ub.es/dsg/2005/ 0503haro.pdf (2005).

[32] J. F. Heagy and S. M. Hammel, The birth of strange nonchaotic attractors, Phys. D, 70 (1994), pp. $140-153$.

[33] M.-R. HeRman, Une méthode pour minorer les exposants de Lyapounov et quelques exemples montrant le caractère local d'un théorème d'Arnol'd et de Moser sur le tore de dimension 2, Comment. Math. Helv., 58 (1983), pp. 453-502.

Copyright ( $\odot$ by SIAM. Unauthorized reproduction of this article is prohibited. 
[34] M. W. Hirsch, C. C. Pugh, And M. Shub, Invariant manifolds, Lecture Notes in Math. 583, SpringerVerlag, Berlin, 1977.

[35] T. H. JÄGER, The creation of strange non-chaotic attractors in non-smooth saddle-node bifurcations, Mem. Amer. Math. Soc., 201 (2009).

[36] A. JoRBA, Numerical computation of the normal behaviour of invariant curves of $n$-dimensional maps, Nonlinearity, 14 (2001), pp. 943-976.

[37] A. Jorba And J. C. TATJER, A mechanism for the fractalization of invariant curves in quasi-periodically forced 1-D maps, Discrete Contin. Dyn. Syst. Ser. B, 10 (2008), pp. 537-567.

[38] L. Kantorovitch and G. Akilov, Analyse fonctionnelle. Tome 2, Mir, Moscow, 1981.

[39] E. W. Kaucher and W. L. Miranker, Self-Validating Numerics for Function Space Problems, Notes and Reports Computer Sci. Appl. Math., Academic Press, Orlando, FL, 1984.

[40] G. Keller, A note on strange nonchaotic attractors, Fund. Math., 151 (1996), pp. 139-148.

[41] J. A. Ketoja And I. I. Satija, Harper equation, the dissipative standard map and strange nonchaotic attractors: Relationship between an eigenvalue problem and iterated maps, Phys. D, 109 (1997), pp. 7080.

[42] H. Koch, A renormalization group fixed point associated with the breakup of golden invariant tori, Discrete Contin. Dyn. Syst., 11 (2004), pp. 881-909.

[43] H. Koch, Existence of critical invariant tori, Ergodic Theory Dynam. Systems, 28 (2008), pp. 1879-1894.

[44] H. Koch, A. Schenkel, And P. WitTwer, Computer-assisted proofs in analysis and programming in logic: A case study, SIAM Rev., 38 (1996), pp. 565-604.

[45] O. E. Lanford, III, A computer-assisted proof of the Feigenbaum conjectures, Bull. Amer. Math. Soc. (N.S.), 6 (1982), pp. 427-434.

[46] O. E. LANFord, III, Computer-assisted proofs in analysis, in Proceedings of the International Congress of Mathematicians, Vols. 1, 2, Berkeley, CA, 1986, American Mathematical Society, Providence, RI, 1987, pp. 1385-1394.

[47] Y. D. LAtushkin And A. M. StËPIn, Weighted shift operators, the spectral theory of linear extensions and a multiplicative ergodic theorem, Mat. Sb., 181 (1990), pp. 723-742.

[48] M. Lerch, G. Tischler, J. W. Von Gudenberg, W. Hofschuster, and W. Krämer, FILIB++, a fast interval library supporting containment computations, ACM Trans. Math. Softw., 32 (2006), pp. 299-324.

[49] U. Lochtelli and A. Giongilli, Invariant tori in the Sun-Jupiter-Saturn system, Discrete Contin. Dyn. Syst. Ser. B, 7 (2007), pp. 377-398.

[50] R. MAÑé, Persistent manifolds are normally hyperbolic, Trans. Amer. Math. Soc., 246 (1978), pp. 261283.

[51] J. N. MAther, Characterization of Anosov diffeomorphisms, Nederl. Akad. Wetensch. Proc. Ser. A 71 Indag. Math., 30 (1968), pp. 479-483.

[52] R. E. Moore, Interval Analysis, Prentice-Hall, Englewood Cliffs, NJ, 1966.

[53] R. E. Moore, Methods and Applications of Interval Analysis, SIAM Studies Appl. Math. 2, SIAM, Philadelphia, 1979.

[54] H. Osinga, J. Wiersig, P. Glendinning, and U. Feudel, Multistability and nonsmooth bifurcations in the quasiperiodically forced circle map, Internat. J. Bifur. Chaos Appl. Sci. Engrg., 11 (2001), pp. 3085-3105.

[55] A. Prasad, S. S. Negi, and R. Ramaswamy, Strange nonchaotic attractors, Internat. J. Bifur. Chaos Appl. Sci. Engrg., 11 (2001), pp. 291-309.

[56] N. Revol, K. Makino, AND M. Berz, Taylor models and floating-point arithmetic: Proof that arithmetic operations are validated in COSY, J. Log. Algebr. Program., 64 (2005), pp. 135-154.

[57] R. J. Sacker, A new approach to the perturbation theory of invariant surfaces, Comm. Pure Appl. Math., 18 (1965), pp. 717-732.

[58] R. J. SaCker And G. R. SEll, Existence of dichotomies and invariant splittings for linear differential systems. I, J. Differential Equations, 15 (1974), pp. 429-458.

[59] J. Stark, Invariant graphs for forced systems, Phys. D, 109 (1997), pp. 163-179.

[60] J. StaRK, Regularity of invariant graphs for forced systems, Ergodic Theory Dynam. Systems, 19 (1999), pp. 155-199.

Copyright (C) by SIAM. Unauthorized reproduction of this article is prohibited. 
[61] R. Sturman and J. Stark, Semi-uniform ergodic theorems and applications to forced systems, Nonlinearity, 13 (2000), pp. 113-143.

[62] W. Tucker, Validated Numerics: A Short Introduction to Rigorous Computations, Princeton University Press, Princeton, NJ, 2011.

[63] D. Wilczak And P. Zgliczyński, $C^{r}$-Lohner algorithm, Schedae Informaticae, 2011.

[64] P. ZgLiczyński, Computer Assisted Proofs in Dynamics, http://www.ii.uj.edu.pl/ zgliczyn/cap07/cap07. htm.

[65] P. ZGLiczyński And M. GideA, Covering relations for multidimensional dynamical systems, J. Differential Equations, 202 (2004), pp. 32-58.

Copyright (c) by SIAM. Unauthorized reproduction of this article is prohibited. 